\title{
Comparative Genomic and Phenotypic Analyses of Pathogenic Fungi Neoscytalidium Dimidiatum and Bipolaris Papendorfii, Isolated From Human Skin Scraping
}

\author{
Chee Sian Kuan \\ Universiti Malaya \\ Kee Peng $\mathrm{Ng}$ \\ Universiti Malaya \\ Su Mei Yew \\ Universiti Malaya \\ Hadiza Umar Meleh \\ Universiti Putra Malaysia \\ Heng Fong Seow \\ Universiti Putra Malaysia \\ Kang Nien How \\ Universiti Putra Malaysia \\ Siok Koon Yeo \\ Taylor's University - Lakeside Campus: Taylor's University \\ Jap Meng Jee \\ Taylor's University - Lakeside Campus: Taylor's University \\ Yung-Chie Tan \\ Codon Genomics Sdn Bhd \\ Wai-Yan Yee \\ Codon Genomics Sdn Bhd \\ Chee-Choong Hoh \\ Codon Genomics Sdn Bhd \\ Rukumani Devi Velayuthan \\ Universiti Malaya \\ Shiang Ling $\mathrm{Na}$ \\ Universiti Malaya \\ Siti Norbaya Masri \\ Universiti Putra Malaysia \\ Shu Yih Chew \\ Universiti Putra Malaysia \\ Leslie Thian Lung Than ( $\square$ leslie@upm.edu.my) \\ Universiti Putra Malaysia https://orcid.org/0000-0001-6956-4638
}

\section{Research article}

Keywords: Dematiaceous, Fungal plant pathogen, Neoscytalidium dimidiatum, Bipolaris papendorfii, Skin scraping

Posted Date: October 28th, 2020

DOI: https://doi.org/10.21203/rs.3.rs-96876/v1

License: (c) (1) This work is licensed under a Creative Commons Attribution 4.0 International License. Read Full License 


\section{Abstract}

Background: Neoscytalidium dimidiatum and Bipolaris species are fungal plant pathogens that have been reported to cause human diseases. Recently, we have isolated numerous $N$. dimidiatum and Bipolaris species from the skin scrapings and nails of different patients. In this work, we have sequenced the genome of one strain of $N$. dimidiatum. The sequenced genome was compared to that of a previously reported Bipolaris papendorfii genome for a better understanding of their complex lifestyle and broad host-range pathogenicity.

Results: Their 33-43 Mb genomes include 11,015-12,320 putative coding DNA sequences, of which 0.51-2.49 are predicted transposable elements. Analysis of secondary metabolism gene clusters revealed several melanin biosynthetic genes and genes involved in fungal iron uptake. The arsenal of CAZymes related to plants pathogenicity is comparable between the species, including genes involved in hemicellulose and pectin decomposition. Several important genes-encoding keratinolytic peptidases were identified in $N$. dimidiatum and $B$. papendorfii, reflecting their potential pathogenic role in causing skin and nail infections. In this study, additional information on the metabolic features of these two species, such as nutritional profiling, $\mathrm{pH}$ tolerance, and osmotolerant are revealed.

Conclusions: The genomic characterization of $N$. dimidiatum and $B$. papendorfii provides the basis for the future functional studies to gain further insights as to what makes these fungi persist in plants and why they are pathogenic to humans.

\section{Introduction}

Neoscytalidium dimidiatum is a dematiaceous hyphomycetes belonging to the order Botryosphaeriales in the class Dothideomycetes. The nomenclature for Neoscytalidium as Neoscytalidium dimidiatum isolate has been a controversial issue due to the production of both arthroconidial (Scytalidium dimidiatum) and pycnidial (Natrassia mangiferae) synanamorphs [1]. Based on the DNA sequence data of 28S rDNA, Crous et al. (2006) reclassified several lineages in the family Botryosphaeriaceae [2]. According to the taxonomic revision, a new genus Neoscytalidium with type species Neoscytalidium dimidiatum to include all isolates that produce arthroconidia typical of S. dimidiatum, including Hendersonula toruloidea and $S$. hyalinum was propose $[1,2,3]$. This revision separated Neoscytalidium dimidiatum from a closely related group (Nattrassia mangiferae) that lacked this typical characteristic [2]. To date, the genus Neoscytalidium contains only two species: N. dimidiatum and N. novaehollandiae.

$N$. dimidiatum is a thermotolerant fungus, which causes sooty cankers on various fruit plants, bark trees and ornamentals, such as physic nut, dragon fruit, orange and common fig in tropical and subtropical regions $[4,5,6,7,8,9]$. Neoscytalidium canker causes wilting and dieback of tree branches when temperature reachs a hot regime and in dry weather condition $[10,11]$. Heat stress turns $N$. dimidiatum into an opportunistic plant pathogen which infects fruit plants and trees $[10,11]$. Neoscytalidium dimidiatum is not only known as phytopathogen but is also notorious for causing onychomycosis [12,13] and dermatophytosis-like skin infection [14]. The diagnosis of such infections is challenging, since $N$. dimidiatum infection is frequently misdiagnosed as dermatophytosis based on direct microscopic examination [15]. Evidence has emerged that this fungus also causes subcutaneous phaeohypomycosis [16], mycetoma [17], cerebral phaeohyphomycosis [18], sinusitis [19], endophthalmitis [20] and fungemia [21], mainly affecting immunocompromised patients.

For the past ten years, the isolation of many dematiaceous fungus, mainly N. dimidiatum and Bipolaris species (teleomorph of Cochliobolus) from the superficial skin and nail of patients of University Malaya Medical Centre (UMMC), a tertiary hospital in Malaysia has been reported. Like $N$. dimidiatum, Bipolaris species are well known as plant pathogens and have been reported to cause devastating disease epidemics of crop plant [21] Bipolaris species has been reported to cause fruit and stem end rot in dragon fruit in Malaysia [22]. In another independent study, a serious stem canker caused by $N$. dimidiatum is found to be infecting most red-fleshed dragon fruit plantation in Malaysia [23]. These suggest an increasing trend of $N$. dimidiatum and Bipolaris species isolation from both plants and humans in Malaysia. Although Neoscytalidium sp. and Bipolaris sp. are morphological different and not closely related phylogenetically [24], they might share similar strategies to colonize and adapt to plant and human hosts.

In this work, we identified and generated a draft genome sequence of $N$. dimidiatum UM 880 that was recovered from the skin scraping of a patient suffering from dermatophytosis. The published Bipolaris papendorfii UM 226 genome [25] enables us to compare the genome sequence assemblies and annotations for $N$. dimidiatum and B. papendorfii. The in-depth comparative analysis of these two pathogens relative to one another and to other related fungal genomes is the first step in understanding their lifestyle and fungal nutritional strategy as well as gain insight into genes that form unique features of their pathogenic success. Knowledge of the basic biology and phenotype of these pathogen are useful in the development of a more suitable strategies for disease management.

\section{Materials And Methods Ethics statement}

The isolates of $N$. dimidiatum UM 880 and B. papendorfii UM 226 used in this study were obtained from an archived fungal collection in a mycology laboratory of a teaching hospital, University of Malaya Medical Centre (UMMC), Kuala Lumpur, Malaysia. All labels on the source sample have been erased with the exception of sample type and clinical diagnosis. Thus, there is no information traceable back to the patient from whom the isolate 
was obtained. In such circumstances, ethical clearance is exempted for this genomic study (http://www.ummc.edu.my/view/content.php? ID=VGxSWIBRPT0=).

\section{Fungal isolate}

Both $N$. dimidiatum UM 880 and $B$. papendorfii UM 226 were isolated from the skin scraping of patients in the UMMC. The isolate was processed according to the laboratory's standard operating procedures (SOP) [24]. The isolate was cultured on Sabouraud Dextrose Agar (SDA) at $30^{\circ} \mathrm{C}$ up to seven days, with alternate day examination for fungal growth. Macroscopic and microscopic examination were carried out as previously described [25].

\section{In vitro antifungal susceptibility}

The in vitro antifungal susceptibility of N. dimidiatum UM 880 and B. papendorfii UM 226 were examined using the Epsilometer Test (Etest, Biomerieux, France) to determine the minimum inhibitory concentration (MIC) of antifungal drugs as previously described [24]. The isolate was tested against eight common antifungal agents (amphotericin B, five azoles, viz. fluconazole, itraconazole, posaconazole, voriconazole, ketoconazole, and two echinocandins, viz. anidulafungin and caspofungin), and the minimum inhibitory concentrations (MICs) were examined after 24-hour incubation.

\section{DNA sequencing and multilocus phylogenetic analysis}

Three different unlinked nuclear loci: ITS, SSU and LSU regions were used to identify the fungal isolate UM 880. Total DNA extraction, PCR amplification, DNA sequencing, and BLASTn search were performed as described previously [24,25]. Unique ITS, SSU and LSU sequences from the isolate um 880, together with an additional species of $N$. novaehollandiae and an outgroup strain of Macrophomina phaseolina (Table S1) were subjected to phylogenetic analysis. Multiple sequence alignments of collected ITS, LSU and SSU sequences were generated using M-Coffee. Individual alignments were concatenated for Bayesian Markov Chain Monte Carlo (MCMC) analysis partitioned by gene. Bayesian tree analyses were performed using MrBayes v3.2.113 with reversible jump MCMC averaging over the entire general time reversible (GTR) rates and gammadistributed rate heterogeneity for all subsets of partitioned scheme. A total of 5,000,000 generations were run with a sampling frequency of 100 , and diagnostics were calculated for every 1,000 generations. The first 12,500 trees were discarded with burn-in setting of $25 \%$. Convergence was assessed with a standard deviation of split frequencies below 0.01 , no obvious trend for the plot of the generation versus the log probability of the data, and a potential scale reduction factor (PSRF) close to 1.0 for all parameters.

\section{Genomic DNA extraction, genome sequencing and assembly}

Genomic DNA extraction was carried out as described previously [25]. Whole genome sequencing of $N$. dimidiatum UM 880 was carried out using Illumina HiSeq 2000 Sequencer (Illumina Inc., San Diego, CA USA) in a 2×90 bp paired-end mode on 500-bp and 5-kb library sizes. Illumina library was prepared using TruSeq v3 Reagent Kits (Illumina Inc., San Diego, CA USA). The selected library fragments were purified through gel electrophoresis, which then selectively enriched and amplified by PCR. The 500-bp Illumina sequenced read was then combined with the 5-kb Illumina sequenced read for further processing. Both sets of sequenced reads were first pre-processed using FASTX-Toolkit (http://hannonlab.cshl.edu/fastx_toolkit/) trimming bases with a Phred quality below Qv20 from the 3'-end of the reads. The trimmed reads shorter than $30 \mathrm{bp}$ and reads with $40 \%$ bases having Qv $\leq 20$ were filtered out, retaining small-insert reads $\geq 80$ bp and large-insert reads $\geq 30$ bp. Two bases were trimmed from the 5 '-terminal of all reads. Pre-processed reads from both libraries were assembled with Velvet version 1.2.07 [26] with kmer setting $=63$, insert length $=503$, -ins_length_sd $=103$, - min_pair_count $=15$, insert_length2 $=5000$, ins_length2_sd $=500$, and min_contig_lgth $=$ 200. Additional parameter of -shortMatePaired = yes was set for large insert library. The generated contig assembled from the Velvet were further scaffolded using SSPACE Basic v2.0 [27] with more stringent parameters than software default to achieve higher accuracy assembly (parameters: - $z$ 100, -k 15, -a 0.3, -n 30 and -T 10). GapFiller v1.10 (-m = 60, $-0=15,-r=0.8,-n=30,-t=30$ and $-\mathrm{T}=10)$ was used to perform gap filling by utilizing paired-end sequencing data from both libraries $[28,29]$.

\section{Genome annotation}

Interspersed repetitive elements and low complexity DNA sequences were masked using RepeatMasker version open-3.3.0 with the Repbase fungal library version rm-20120418, followed by masking off the RNA sequences. Individual rRNA and tRNA were predicted using RNAmmer v1.2 [30] and tRNAscan-SE v1.3.1 [31], respectively. Putative transposable elements were identified using Transposon-PSI (http://transposonpsi.sourceforge.net) by PSI-TBLASTN searches with a collection of (retro-) transposon open reading frame (ORF) homology profiles. Protein coding sequences were predicted using GeneMark-ES version 2.3e [32]. Gene annotation was completed using BLAST (Basic Local Alignment Search Tool) searches against the NCBI nr and SwissProt databases.

Page 3/32 


\section{Functional gene and pathway analysis}

GO and KEGG metabolic pathways matches were performed using local BLAST2GO tools [33]. Classification of predicted proteins were carried out using KOG [34]. Protein domain families were matched to Pfam database using InterProScan 5 [35]. The best protein models of the $N$. dimidiatum UM 880 and B. papendorfii UM 226 genomes were submitted to database of automated Carbohydrate-active enzyme ANnotation (dbCAN) to functionally annotate CAZymes. Comparative analysis was performed against other fungi with different lifestyles, including saprophytic fungi (Neurospora crassa OR74A and Trichoderma reesei QM6a), facultative parasitic fungi (Aspergillus nidulans FGSC A4), biotrophic fungi (Cladosporium flavum, Ustilago maydis, and Blumeria graminis), necrotrophic fungi (Cochliobolus heterostrophus $\mathrm{C} 4$ and $C$. heterostrophus $\mathrm{C5}$ ), hemi-biotrophic fungi (Cochliobolus sativus ND90Pr, Magnaporthe oryzae 70-15 version 8, and Fusarium graminearum PH-1), and symbiotic fungus (Laccaria bicolor) [36,37]. Peptidases were functionally annotated by submitting the predicted protein models to the MEROPS database [38]. SignalP version 4.1 [39] was used to predict cleavage sites and signal peptide/non-signal peptide to identify small secreted proteins after discarding proteins with transmembrane domains that were determined by TMHMM version 2.0 [40]. One transmembrane domain located at the $\mathrm{N}$-terminal 40 amino acids is acceptable as the domain is responsible to the secretion signal. Genomic mapping of fungal secondary metabolite backbone genes and associated genes was carried out using web-based SMURF (Secondary Metabolite Unknown Regions Finder) (www.jcvi.org/smurf/) [41]. Amino acid sequences with e-value threshold $\leq 1 \mathrm{e}-5$, alignment length over $70 \%$ of its own length and over $50 \%$ match identity were selected and assigned as the annotation of predicted genes.

\section{Phenotype microarrays}

Biolog PM analysis (Biolog Inc., Hayward, CA, USA) was employed to examine the metabolic profile of Bipolaris papendorfii and Neosyntalidium dimidiatum. Ten MicroPlate panels (PM1 to PM10), which included carbon, nitrogen, phosphorus, sulfur, nutrient supplements, peptide, osmolytes, and $\mathrm{pH}$ sources, were used in this study as summarized in Table S13. Bipolaris papendorfii and Neosyntalidiumdimidiatum were grown on SDA at $30^{\circ} \mathrm{C}$ for 7 days. Colonies on SDA were extracted with sterile, wetted cotton swab and inoculated into $15 \mathrm{~mL}$ of yeast solution (Biolog Inc.). The turbidity of the cell suspension was adjusted to $62 \%$ transmittance at $590 \mathrm{~nm}$. PM1-10 inoculating fluid were prepared according to the manufacturer's protocol. The cell suspension was inoculated to PM1-10 inoculating fluid as indicated in Table S14. The prepared inoculum suspension $(100 \mu \mathrm{L})$ was inoculated into each wells of microplate panel PM1-10 and incubated at $30^{\circ} \mathrm{C}$ for 120 hours. Sample were done in duplicates and readings were taken using Epoch Microplate Spectrophotometer (BioTek Instruments, Inc., Winooski, VT, USA). The utilization of substrate was monitored at $490 \mathrm{~nm}$ in PM 1-8 microplate while the growth or biomass reaction to osmolytes and pH were monitored at $750 \mathrm{~nm}$ in PM 9-10 microplate. Water was used as the negative control for each microplate. Data for each assay from duplicate runs were averaged to obtain the mean value.

\section{Statistical analyses}

Data were evaluated using SPSS Inc. software (version 20) (SPSS Inc., Chicago, USA). All data are presented as mean from two separate runs ( $\mathrm{n}=$ 2 ), unless stated otherwise. The statistical difference between sample means for analyses involving supplementation of nitrogenous source at $\mathrm{pH}$ 4.5 and 9.5 as well as supplementation of osmolyte at $6 \%$ sodium chloride was analysed with One Way ANOVA. The multiple comparison of means were analysed by Tukey's test. An independent T-test was used to determine the significant difference between means for $\mathrm{pH}$, salt and urea tolerance test. The statistical level of significance was preset at $a=0.05$.

\section{Results And Discussion}

\section{Identification of $\mathbf{N}$. dimidiatum UM 880}

The $N$. dimidiatum UM 880 colonies on Sabouraud dextrose agar (SDA) were fast growing with dense, deeply floccose and cottony aerial mycelium (Figs $1 \mathrm{~A}$ and $1 \mathrm{~B}$ ). The colony reached diameters of $90 \mathrm{~mm}$ and filling the petri within 4 to 6 days at $30^{\circ} \mathrm{C}$ (Fig $1 \mathrm{~A}$ ). The surface of the colonies was initially greyish white, becoming dark grey and produced blackish brown diffusible pigment within 6-days incubation (Fig 1A). It had a black coloration on the reverse side (Fig 1C).

Microscopic examination of slide culture preparations demonstrated broad branched, septate, subhyaline to dark brown hyphae (6-10 $\mu$ m wide) with no conidiophores (Fig 1D). They are constricted at their prominent thick septations, giving the pseudohyphae appearance. Contiguous of one- to twocelled, pigmented arthroconidia (3-6 × 5-12 $\mu \mathrm{m})$ with rectangular, oval and barrel-shaped were produced (Fig 1D). The arthroconidia were flattened on the ends; two-celled conidia were separated by a thick septum (Fig 1D). No pycnidium was produced by UM 880. The SEM microphotograph of the UM 880 revealed that the arthroconidia were cylindrical with smooth to slightly verruculose walled and arranged in chains. The end of each conidium was central convex dome in shape and was connected to other conidium. Based on the typical morphological features as described previously [42] the UM 880 clinical isolate was identified as $N$. dimidiatum.

Page 4/32 
Molecular identification was performed to confirm that the UM 880 isolate is truly $N$. dimidiatum as suggested by its morphological features. The internal transcribed spacer region (ITS), small subunit of the ribosomal RNA gene (SSU) and large subunit of the ribosomal RNA gene (LSU) sequences were obtained by amplification and bidirectional sequencing. PCR amplification of the ITS, LSU and SSU gene regions yielded specific amplicons of approximately $560 \mathrm{bp}$ (ITS), 1,200 bp (SSU) and 2000 bp (LSU), respectively (Fig S1). By querying ITS, LSU and SSU nucleotide sequences against those deposited in the NCBI-nucleotide database, the sequenced ITS gave $100 \%$ query coverage and $100 \%$ (579/579) identity with $N$. dimidiatum strain CBS 499.66; the sequenced LSU gave $72 \%$ query coverage and $100 \%(1159 / 1159)$ identity with $N$. dimidiatum strain CBS 145.78; the sequenced SSU gave $100 \%$ query coverage and $100 \%$ (1024/1024) identity with $N$. dimidiatum strain CMW 26170 . The sequenced ITS, LSU and SSU gene regions were used to construct a phylogram using combined gene analysis with the additional 16 ex-type strains of Neoscytalidium species. (Fig 2 and Table S1). Multilocus phylogenetic analysis revealed that the UM 880 isolate was clustered together with the group of $N$. dimidiatum instead of being grouped together into $N$. novaehollandiae cluster (Fig 2). Taken together, the morphological and molecular characterisation of UM 880 isolate revealed that it is $N$. dimidiatum.

\section{Properties of the $\boldsymbol{N}$. dimidiatum and $B$. papendorfii genomes}

The $N$. dimidiatum UM 880 genome was sequenced to 93-fold depth of genome sequence coverage. In this study, a total of 33,078,316 paired reads $(3.3 \mathrm{~Gb})$ of a 500-bp insert-size library and 10,699,352 paired reads (1.1 Gb) of a 5-kb insert-size library were generated by Illumina HiSeq 2000 Sequencing system. The assembly yielded a combined total length of $42,687,746$ bases in 2,230 contigs ( $\geq 200$ bases) which were then placed into 110 scaffolds ( $\geq 1,000$ bases) with paired-end sequencing data from both libraries. The $B$. papendorfii UM 226 genome was generated by a hybrid assembly of Illumina short reads and PacBio long reads with a lower number of contigs and a higher N50 value (Table 1). The larger genome for $N$. dimidiatum UM 880 genome ( 43 Mb) as compared to the $B$. papendorfii UM 226 genome ( 33 Mb) [25] is most likely due to the lower sequence coverage, as small scaffolds may fall into larger scaffold gaps. The $\mathrm{G}+\mathrm{C}$ content of $N$. dimidiatum UM 880 (54.16\%) was comparable to $B$. papendorfii UM 226 (50.65). Overall, the summary of the sequence statistics was shown in Table 1.

\section{Transposable elements}

Transposable elements (TEs) are ubiquitous components in fungal genomes. Transposable elements play an important role in the genetic variation and actively affect gene structure to allow them to adapt in adverse environments, especially for fungus that lacks sexual stages [43]. Dematiaceous fungal genomes contain different amounts of TEs. For example, the assembled genome of $B$. papendorfii UM 226, Cochliobolus heterostrophus race O genome, Sporothrix schenckii, and Sporothrix brasiliensis contain 2.49\%, 5.90\%, 0.34\%, and 0.62\% of TEs, respectively [25,44,45]. Transposable elements identified in N. dimidiatum UM 880 and B. papendorfii UM 226 genomes were categorised into classes and annotated as class I (LTR and LINE) and class II transposons. The assembled genome of $N$. dimidiatum UM 880 contains low TEs content ( $0.51 \%)$, whereas these "jumping genes" occupy $2.49 \%$ of the $B$. papendorfii UM 226 genome (Table 2). The high TEs content in B. papendorfii UM 226 might impact on its genome organization, evolution and genomic functions, including gene silencing, regulation of gene expression or gene mutation. Despite the smaller genome size of the $B$. papendorfii UM 226, it has a 4-fold higher TEs content, and has either class I (retrotransposons) or class II DNA transposons as compared to $N$. dimidiatum UM 880. LINE (Long Interspersed Nuclear Element) and LTR (Long Terminal Repeat) were mostly identified in the $N$. dimidiatum UM 880 genome (Table 2), which is common for fungal genomes [45,46]. Bipolaris papendorfii UM 226, however, contains high copy number of DDE_1 transposases, which contain functional domains to mediate DNA-binding, protein-protein interaction and "cleavage-and -joining" activities [46]. Gypsy element is the LTR superfamily that is widely distributed in the genome. A total of 83 and 279 copies of Gypsy elements were identified in N. dimidiatum UM 880 and B. papendorfii UM 226 genomes, respectively. Santana et al. (2014) reported that an estimated 50\% of TEs identified in the $C$. heterostrophus race $O$ genome was Gypsy elements [45]. Gypsy elements were found to be located in the non-coding regions adjacent to the PKS1, PKS2, OXI1, LAM1, RED2 and RED3 genes, but their exact function remains unknown [45]. No SINE (Short Interspersed Nuclear Elements) retrotransposon was identified in neither $N$. dimidiatum UM 880 nor $B$. papendorfii UM 226 genomes.

\section{Gene content}

A total of 137 tRNAs and 23 rRNAs (20 8S/5.8S rRNA, one 18S rRNA, and two 28S rRNA) were identified in the $N$. dimidiatum UM 880 genome whereas B. papendorfii UM 226 genome contains 128 tRNAs, 12 5S rRNA, three 18 S rRNA, and three $28 \mathrm{~S}$ rRNA. The $N$. dimidiatum UM 880 genome revealed a total of 12,320 putative coding DNA sequences (CDS) with gene density of 2.88 genes/10 kb. The assembly size and the total number of predicted genes in the $N$. dimidiatum UM 880 genome were compared to other dematiaceous fungi. Neoscytalidium dimidiatum UM 880 has a relatively larger genome but lower gene density compared to other dematiaceous fungi (Table S2), reflecting larger amounts of non-coding DNA in the UM 880 genome. On average, there were 3.09 exons per gene and average size of protein coding genes was 1,451 bp in $N$. dimidiatum UM 880 genome. In addition, $N$. dimidiatum UM 880 genome revealed that a total of 11,577, 7,656 and 9,155 gene-coding sequences were homologous to known proteins deposited in the NCBI nr, SwissProt and InterPro databases, and are comparable to that of B. papendorfii UM 226 (Table 3 and S3).

Hypothetical proteins are predicted proteins, which are characterized by low identity to annotated proteins and have not been functionally characterized [47]. Surprisingly, both N. dimidiatum UM 880 and B. papendorfii UM 226 genomes harbor large amount of hypothetical proteins or

Page 5/32 
unannotated proteins. The $N$. dimidiatum UM 880 and B. papendorfii UM 226 genomes contain a total of 6,041 and 10,330 hypothetical proteins/ unannotated proteins but lacked significant homologies to known proteins from NCBI nr database. (Table S4). InterPro protein sequence analysis revealed at least one domain was identified in 3,098 and 7,614 hypothetical proteins/ unannotated proteins in $N$. dimidiatum UM 880 and $B$. papendorfii UM 226 genomes, respectively (Table S4). Most of the hypothetical proteins and unannotated proteins contain protein kinase domain (169 and 290 in N. dimidiatum UM 880 and B. papendorfii UM 226 genomes, respectively), suggesting the importance of these unknown proteins involved in phosphorylation to govern various cellular processes, such as metabolism, transcription, cell movement, apoptosis, and differentiation by changing cellular location, enzyme activity, or association with other proteins.

The $N$. dimidiatum UM 880 and B. papendorfii UM 226 genomes were also mapped to Eukaryotic Clusters of Orthologs (KOG), Gene Ontology (GO), and the Kyoto Encyclopedia of Genes and Genomes (KEGG) databases to characterize the predicted proteins. In $N$. dimidiatum UM 880 genome, a total of 6,182 proteins were ascribed to 26 different functional groups based on KOG classification (Fig 3A and Tale S5). The category [R] General functions prediction only contains the most number of genes (1,234 genes), shows that many proteins were not classified to a distinct group (Fig 3A). The categories [0] Post-translational modification, protein turnover, chaperones (542 genes), [Q] Secondary metabolites biosynthesis, transport and catabolism (539 genes), [1] Lipid transport and metabolism (467 genes), [C] Energy production and conversion (438 genes) and [T] Signal transduction mechanisms (423 genes) were the top five most abundant KOG groups (Fig3A). Neoscytalidium dimidiatum UM 880 and B. papendorfii UM 226 genomes contain a large fraction of putative genes that are associated with post-translational modifications, protein turnover, and chaperones (Fig 3A). Post-translational modifications of fungal cell surface proteins, such as reversible peptide modifications ubiquitination, neddylation, and sumoylation are vital in fungal pathogenicity [48]. The putative genes that are related to ubiquitination, neddylation, and sumoylation are listed in Table S6.

Gene ontology (GO) analysis revealed that the N. dimidiatum UM 880 and B. papendorfii UM 226 have 7,202 and 7,155 genes with at least one G0 assignment, respectively. All genes that matched to "Cellular Component", "Molecular Function", and "Biological Process" categories of GO (Table 4) was further examined. A 0.05 p-value of Pearson Chi-Square test is used as the cut off for the significance of the relationship between the two datasets. The relationship between the two species is considered remarkable when the p-value is below this significant level. In the "Cellular Component" ontology, we found that genes that mapped to the cell (G0:0005623) and organelle (G0:0043226) were significant higher in $\mathrm{N}$. dimidiatum UM 880 as compared to the B. papendorfii UM 226. In the "Molecular Function" ontology, N. dimidiatum UM 880 contains higher number of genes involved in transporter activity but lower number of genes involved in binding activity. The higher transporter activity might compensate the low binding activity in acquisition of molecules such as vitamins and cofactors in UM 880. UM 880 and UM 226 are thus suggested to employ different strategies to regulate molecule movements. In the "Biological Process" ontology, UM 880 contains more genes assigned to the "response to stimulus" (G0:0050896) category but lesser genes in the "metabolic process" (G0:0008152) category than UM 226. In the "metabolic process" category, the "nitrogen compound metabolic process" (G0:0006807), "macromolecule metabolic process" (G0:0043170), "primary metabolic process" (G0:0044238), and "oxidation reduction" (G0:0055114) are categories which showed remarkable relationship. In contrary, significant relationship was further noticed in the category of "response to chemical stimulus" (G0:0042221), under the subset of "response to stimulus". Of the 1,110 genes assigned to this category in UM 880, 201 genes were further assigned to the "response to drug" (G0:0042493) category (168 genes in UM 226), 274 genes into "response to xenobiotic stimulus" (G0:0009410) category (237 genes in UM 226) and 246 genes to "response to inorganic substance" (G0:0010035) category (207 genes in UM 226). Particularly, the genes assigned to "drug transport" (G0:0015893) and "multidrug transport" (G0:0006855) are markedly higher in UM 880 (78 and 26 genes, respectively) than in UM 226 (39 and 28 genes, respectively). This might contribute to the higher tolerance of drugs in UM 880 as compared to UM 226 as will be discussed in the section of "Antifungal susceptibility profile and drug resistance genes".

To further gain insight into the gene functions in $N$. dimidiatum UM 880, we successfully assigned 1,812 predicted proteins to their orthologous genes in metabolic pathways in the KEGG database (Table S7). The top five categories in KEGG metabolic pathway in both fungi are carbohydrate metabolism, amino acid metabolism, lipid metabolism, nucleotide metabolism, and xenobiotics biodegradation and metabolism (Fig3B). Overall, $B$. papendorfii UM 226 has noticeably more KEGG annotated genes involved in the metabolic pathway as compared to the $N$. dimidiatum UM 880 (Fig 3B). However, it should be noted that the numbers of genes encoding enzymes for secondary metabolite biosynthesis are more numerous in $N$. dimidiatum UM 880 than in B. papendorfii UM 226 (Fig 3B), which is in consistent with KOG annotation (Fig 3A). The metabolism and biosynthesis of secondary metabolites in N. dimidiatum UM 880 and B. papendorfii UM 226 are further discussed in the subsection of "Secondary metabolism".

\section{Secondary metabolism}

Armed with the knowledge that Dothideomycetes fungi harbour large number of genes encoding enzymes for secondary metabolite production, including polyketide synthase (PKS) and non-ribosomal peptide synthetase (NRPS) that are vital in niche exploitation as well as pathogenicity $[49,50]$. Many of these secondary metabolites are served as virulence factors, which required for host specificity of necrotrophs [51,52]. With this in mind, we screened the UM 880 and UM 226 genomes to identify secondary metabolites that are important for virulence and survival such as host specific toxins (HST), non-host specific toxin (NHST) and melanin. Secondary Metabolite Unique Regions Finder (SMURF) analysis predicted 39 and 32 gene clusters encoding backbone enzymes in N. dimidiatum UM 880 and B. papendorfii UM 226 genomes, respectively (Table 5). 
Dematiaceous fungi are remarkable organisms to produce melanins during formation of fungal spore for deposition in the cell wall. Fungal melanins are essential for survival, pathogenicity, and increase in their competitive ability to occupy plethora of niches. Jahn et al. (2000) indicated that loss of melanin pigment is associated with enhanced susceptibility to reactive oxygen species (ROS) [53]. Most fungal melanins are biosynthesized via 1,8-dihydroxynaphthalene (DHN) pathway using polyketide synthases (PKs) to form melanin precursors. The PKSs are encoded by the most widely distributed fungal PKS genes, such as PKS1 in Colletotrichum lagenarium [50] and PKS18 in Cochliobolus heterostrophus [54]. In this study, PKS1 (UM880_8812, 70.2\% identical), PKS15 (UM880_2257, 51\% identical), alternapyrone synthase, PKSN (UM880_2826, 56\% identical), and PKS13 (UM880_9993, 53\% identical) were identified in N. dimidiatum UM 880 genome; PKS1 (UM226_1411, 93\% identical), PKS2 (UM226_3388, 84\% identical), PKS4 (UM226_8230, 79\% identical), PKS7 (UM226_6241, 65\% identical), PKS9 (UM226_7130, 77\% identical), and PKS11 (UM226_7830, 85\% identical) were found in B. papendorfii UM 226 genome. Like B. papendorfii UM 226 [25], N. dimidiatum UM 880 may produce 1,8-dihydroxynaphthalene (DHN) melanin by a set of DHN melanin-synthesis related proteins, including PKS1, 1,3,6,8-tetrahydroxynaphthalene reductase (BRN2) (UM880_8808, 90\%), transcription factor CMR1 (UM880_8809, 94.1\%), and three scytalone dehydratases (UM880_2773, 85\%, UM880_3280, 98.9\%, and UM880_7245, 50.3\%). Small gene cluster comprising PKS1 BRN2, and CMR1 was identified in N. dimidiatum UM 880 genome (Fig4A). The mutual orientation of PKS1, BRN2, and transcription factor CMR1 is the same as found in B. papendorfii UM 226 (Fig 4A). Amino acid alignment revealed that $N$. dimidiatum UM 880 PKS1 (NDPKS1) is 70.7\% identical to B. papendorfii UM 226 PKS1 (BPPKS1). Both the NDPKS1 and BPPKS1 contain features of a non-reducing fungal type I PKS with a starter unit of ACP transacylase (SAT), $\beta$-ketoacyl synthase (KS), acyltransferase (AT), product template (PT), acyl carrier protein (ACP), and thioesterase (TE) [55]. NDPKS1 and BPPKS1 contain domain order of KSAT-ACP-ACP-TE similar to Bipolaris oryzae PKS1 that is involved in melanin biosynthesis [56] (Fig 4B). Neoscytalidium dimidiatum UM 880 contains two mitogen-activated protein kinases, Chk1 (UM880_5115, 91.7\%) and Mps1 (UM880_607, 86\%) that play important role to regulate transcription factor CMR1 at the transcriptional level [54].

The 6-methylsalicylic-acid synthase belongs to a class of PKSs that is involved in building of an aryl moiety in many bioactive secondary metabolites, such as avilamycin, calicheamicin cholorothricin, maduropeptin, pactmycin, and polyketomycin [57]. In this work, we found a gene encoding 6-methylsalicylic-acid synthase (UM880_11018, 85.2\%) in UM 880. As described in Ding et al. (2010), UM880_11018 harbors multiple functional domains in an order of KS-AT-dehydratase (DH)-ketoreductase (KR)-ACP [57]. The presence of this encoding gene in UM 880 suggests that this isolate may produce a wide array of antibiotics with antitumor activity.

\section{Iron uptake and homeostasis}

Fungal pathogens are capable of acquiring iron from vertebrate hosts in order to survive and reproduce in the hosts. Additionally, iron acquisition is an important infection process for fungi [58]. Fungi commonly use three high-affinity iron uptake mechanisms: i.) nonribosomally synthesized secreted iron chelators (siderophores) ii.) non-siderophore reductive iron assimilation (RIA) and iii.) direct Fe ${ }^{2+}$ uptake to regulate iron homeostasis $[59,60,61,62]$. Fungal siderophores are synthesized by multi domains NRPSs which are encoded by NRPS2 (its homologue SidC in Aspergillus fumigatus) and NRPS6 (its homologue SidD in Aspergillus fumigatus) [60]. In this study, we identified ferrichrome-type NRPS, NRPS2 (UM880_724) and fusarinine-type NRPS, NRPS6 (UM226_3398) in UM 880 and UM 226, respectively. This finding revealed that UM 880 employs the intracellular siderophore, ferricrocin, but UM 226 uses the extracellular siderophore, fusarinine $\mathrm{C}$ for iron acquisition. NRPS6 is a virulence factor for Fusarium graminearum, Cochliobolus heterostrophus, C. miyabeanus, and A. brassicicola [60]. Knockdown of the gene in these fungi caused a significant reduction in virulence and hypersensitive to oxidative stress $[60,63]$. Here, we showed that UM226_3398 is a pathogenicity-associated gene predicted by the pathogen-host interaction database (PHI-base). However, NRPS2 encoded ferricrocin plays no role in virulence of $C$. heterostrophus, but is required for sexual development [63]. In addition, two genes encoding L-ornithine- $\mathrm{N}^{5}$-monooxygeneses were found in UM 880 (UM880_818 and UM880_8078) and UM 226 (UM226_2340 and UM226_3401). The L-ornithine- $\mathrm{N}^{5}$-monooxygenese catalyse the committed step for the biosynthesis fusarinine $\mathrm{C}$ and ferricrocin.

Most fungi employ more than one iron-uptake mechanism in iron homoeostasis. Aspergillus fumigatus employs siderophore and RIA mechanism to acquire iron [62] Our findings showed that both UM 880 and UM 226 not only harbor siderophore to acquire iron but also contain all essential genesencoding enzymes involved in the three-step RIA process. In UM880, we found a metalloreductase Fre1p (UM880_12166), four ferroxidases Fet3 (UM880_431, UM880_937, UM880_2901, and UM880_11949), and two iron permease Ftr1 (UM880_2900 and UM880_432) whereas a metalloreductase Fre8 (UM226_5148), two ferroxidases Fet3 (UM226_875 and UM226_5495), and an iron permease Ftr1 (UM226_876) were identified in UM 226. As previously reported [64,65], ferroxidase and iron permease encoding gene clusters were determined in both UM 880 and UM 226. In UM 880 and UM 226, the iron permeases Ftr1 were located adjacent to ferroxidases Fet3.

\section{Genomes of $\boldsymbol{N}$. dimidiatum and B. papendorfii are particularly suited for hemicellulose and pectin decomposition}

The plant cell wall is composed of polysaccharides cellulose, hemicellulose (composed of xyloglucans, xylans, mannans, glucomannans, and betaglucan), and pectin (composed of galacturonan and rhamnogalacturonan). The capability to breakdown plant cell wall carbohydrates for nutrient sources or facilitate infection via host-pathogen interactions are important features of fungal lifestyle. Fungi can produce various carbohydrate-

Page $7 / 32$ 
active enzymes (CAZymes) to degrade complex plant polysaccharide materials to simple monomers for carbon sources [37]. The abundant of fungal CAZymes demonstrate a remarkable functional diversity and fungal lifestyle due to the vast structural and functional diversity of the complex network of different plant cell wall polysaccharides. The $N$. dimidiatum UM 880 genome was mapped to the CAZy database to identify the presence of CAZyme-coding homologous genes. The CAZyme content in the genome of N. dimidiatum UM 880 was compared to B. papendorfii UM 226 and other fungi from different nutritional modes or infection mechanisms to determine their digestive potential against various plant cell wall polysaccharides. The lifestyle of $N$. dimidiatum and B. papendorfii was determine by comparing their CAZyme content and distribution to saprophytic fungi (Neurospora crassa OR74A and Trichoderma reesei QM6a), facultative parasitic fungi (Aspergillus nidulans FGSC A4), biotrophic fungi (Cladosporium flavum, Ustilago maydis, and Blumeria graminis), necrotrophic fungi (Cochliobolus heterostrophus $\mathrm{C} 4$ and $C$. heterostrophus C5), hemi-biotrophic fungi (Cochliobolus sativus ND90Pr, Magnaporthe oryzae 70-15 version 8, and Fusarium graminearum $\mathrm{PH}-1$ ), and symbiotic fungus (Laccaria bicolor). The N. dimidiatum UM 880 genome contain 669 genes encoding putative CAZymes, comprising 68 carbohydrate binding module (CBM), 162 carbohydrate esterases (CE), 305 glycoside hydrolases (GH), 104 glycosyl transferases (GT) and 30 polysaccharide lyases (PL) (Table S8). At the first glance, the CAZyme content in N. dimidiatum UM 880 and B. papendorfii UM 226 genomes are larger than saprotrophs, biotrophs, and symbiotic fungi but comparable to other plant pathogens (necrotrophs and hemibiotrophs) (Fig 5A and Table S8). In general, plant pathogenic fungi contain more CAZymes than saprotrophic, biotrophic, and symbiotic fungus [36]. The high number of CAZymes in N. dimidiatum UM 880 and B. papendorfii UM 226 genomes suggesting their capability in degrading plant carbohydrates to access internal plant tissues and causing infection in plants.

CAZyme catalytic domains of $\mathrm{CE}, \mathrm{GH}$, and PL are well known as cell wall degrading enzymes [66]. The genomes of $N$. dimidiatum UM 880 and $B$. papendorfii UM 226 contain 196 and 186 CAZymes unambiguously associated with the degradation of plant cell wall polysaccharides cellulose, hemicellulose, pectin as well as hemicellulose or pectin side chains (Table S9). The number of plant cell wall degrading enzymes in $N$. dimidiatum UM 880 and B. papendorfii UM 226 genomes was not far-off to plant pathogenic fungi but higher than in the other non-pathogenic fungi analysed except for $C$. fulvum (Fig 4B). As previously noted [37], C. fulvum differs from other biotrophs, which contains more plant cell wall degrading enzymes families of $\mathrm{GH} 28, \mathrm{GH} 31, \mathrm{GH} 35, \mathrm{GH} 43, \mathrm{CE} 5$, and CE16 (Table S9). Besides the quantitative similarity, the $N$. dimidiatum UM 880 shows qualitative similarity to the $B$. papendorfii $U$ M 226, especially, the cellulolytic and hemicellulolytic capacity as well as the cleavage activity of hemicellulolytic and pectin side chains (Fig 4B and Table S5). The number of putative cellulose degrading enzymes encoded in $N$. dimidiatum UM 880 and $B$. papendorfii UM 226 is smaller than in plant cell wall degrading pathogens (necrotrophs and hemibiotrophs), indicating that they do not extensively digest cellulose. However, their potential cellulose degrading activity is comparable to that of saprotrophic, symbiotic and facultative parasitic fungi (Table S9). In particular, biotrophic fungi have a low cellulose degrading capacity as compared to that of $N$. dimidiatum UM 880 and B. papendorfii UM 226. The members of GH61 family are copper-dependent lytic polysaccharide monooxygenases that play important role to enhance lignocellulose degradation by catalyzing the oxidative cleavage of cellulose [67]. However, the GH61 was not identified in both $N$. dimidiatum UM 880 and B. papendorfii UM 226, whereas necrotrophic and hemibiotrophic fungi have an average of 22 genes (minimum 15 , maximum 25). Biotrophic, saprophytic, and facultative parasitic fungi contain lower number of GH61 CAZymes. The hemicellulose degrading capacity is significantly larger for $N$. dimidiatum UM 880 and B. papendorfii UM 226 than in other plant cell wall degrading fungi (Fig 5B and Table S9). The unique feature of the $N$. dimidiatum UM 880 and $B$. papendorfii UM 226 is the presence of many CE1 proteins (31 and 42 , respectively), whereas other analyzed fungi have an average of four genes (minimum 0 , maximum 8) (Table S9). Both $N$. dimidiatum UM 880 and $B$. papendorfii UM 226 contain respectively two and one CE1 acetyl xylan esterase that directed against the plant cell wall xylan. The two xylanase families GH10 and $\mathrm{GH} 11$, however, equivalent in size to other fungi, except for L. bicolor and B. graminis, which lack of any families GH10 and GH11.

Neoscytalidium dimidiatum UM 880 contain a noticeably higher number of CAZymes putatively involved in pectin degradation as compared to the $B$. papendorfii UM 226 (64 vs. 38 putative pectin-specific CAZymes, respectively) and other fungi (Table S9). The pectin degrading capacity for to $B$. papendorfii UM 226 is comparable to that of necrotrophic and hemibiotrophic fungi in the number of enzymes in all pectin-related CAZY families as well as the ratio of pectin-degrading lyases versus hydrolases, except for M. oryzae 70-15 version 8 (Table S9). Neoscytalidium dimidiatum UM 880 has significantly higher number of polygalacturonases (family GH28), pectin methylesterases (family CE8), and pectate lyases (families PL1 and PL3) than in all the other plant cell wall degrading fungi (Table S9), suggesting that the fungus has high capacity of pectin degradation. The observations also propose a common preference for soft plant tissues, such as fruits or flowers that are rich in pectin. Different plant cell wall degrading fungi likely employ different methods to degrade plant tissues. In this work, the results suggest a preference of $N$. dimidiatum UM 880 and B. papendorfii UM 226 for hemicellulose and pectin rather than for cellulose. Although the cellulose degrading capacity is similar between them, their ability to degrade soft tissue components, especially pectin is much different. Even if it lacks experimental supports, the lower number of cellulose degrading enzymes in both $N$. dimidiatum UM 880 and B. papendorfii UM 226 suggesting that they employ another strategy to digest cellulose.

\section{Secreted peptidases}

Dermatophytes harbor the ability to secrete peptidases to degrade compact hard keratin for carbon and nitrogen sources. At this stage of knowledge, $N$. dimidiatum and B. papendorfii are not known as dermatophytes. However, the isolation of UM 880 and UM 226 from the human skin lesions had raised our interest to search for genes encoding keratin degradation proteases in these genomes. In line with this information, peptidase-encoding genes were classified in the $N$. dimidiatum UM 880 and B.papendorfii UM 226 genomes using the MEROPS database, with emphasis on the secreted peptidases (Table S10). Neoscytalidium dimidiatum UM 880 and B. papendorfii UM 226 genomes contain 199 and 153 peptidases, respectively. Of

Page $8 / 32$ 
these, a total of 58 and 35 of the deduced peptidases containing a secretion signal in UM 880 and UM 226 genomes, respectively. This preliminary data showed that UM 880 possesses greater metabolic capability for protein degradation as compared to UM 226. Burmester et al. (2011) revealed that keratinolytic peptidases belong to the families M14 (metallocarboxypeptidases), M28 (aminopeptidases), M35 (deuterolysin), M36 (fungalysin), S8 (subtilisin), and S10 (carboxypeptidases) have expanded in dermatophytes Arthroderma benhamiae and Trichophyton verrucossum [68]. In this study, collections of predicted secreted peptidases in M14, M28, M35, M36, S8, and S10 families of UM 880 and UM 226 were compared to those A. benhamiae and T. verrucossum (Table 6). UM 880 contains lower number of peptidases family S8, S14, and M28 while UM 226 contains lesser peptidases family S8 and S10 as compared to A. benhamiae and T. verrucossum. No deuterolysin was found in UM 226 . Additionally, fungalysin was not identified in both UM 880 and UM 226.

The data was further analyzed by searching into the known keratinolytic peptidases in UM 880 and UM 226 genomes based on literature review. Here, we managed to identify a total of potential 13 and 18 keratinolytic peptidases in UM 880 and UM 226 genomes, respectively (Table 7). Of the secreted peptidases, one gene (UM880_9235) in UM 880 and two genes (UM226_975 and UM226_2139) showed 46\%, 51\%, and 45\% identity to T. rubrum metallopeptidase TruMCPA (ABW79919), respectively. Multiple sequence alignment was constructed and the conserved catalytic sites, zinc binding, substrate binding and cysteine residues were identified (Fig S2). Two genes in N. dimidiatum, UM880_7307 and UM880_4501 were identified as putative leucine aminopeptidase LAP1 and LAP2 with 49\% and 46\% identity to T. rubrum LAP1 and LAP2, respectively. Whereas in $B$. papendorfii, a total of six putative aminopeptidases were determined, comprising two putative LAP1 (UM226_9084 and UM226_9684) and three putative LAP2 (UM226_5777, UM226_3516, UM226_7154 and UM226_10463). The hydrolytic activities of both LAP1 and LAP2 peptidases are the same [69]. The consensus active residues and binding sites of these predicted peptidases are the same as reported [69] (Figs S3 and S4).

Additionally, the S9 family dipeptidyl peptidases (DPPs) were identified in both genomes. Both genomes contain a gene encoding DPPIV with $61 \%$ similar to T. rubrum and $A$. fumigatus DPPIV in UM 880 (UM880_10172) and; 56\% and 57\% identity similar to T. rubrum and A. fumigatus DPPIV in UM 226 (UM226_84). However, UM 226 has two genes more than UM 880 that encode DPPV. The consensus motif catalytic triad of the putative DPPIVs and DPPVs were identified in the multiple sequence alignments (Figs S5 and S6).

We also identified three secreted peptidases each from UM 880 and UM 226 that shared similarity to serine carboxypeptidases (S10) associated with keratin degradation in T. rubrum (TruSCPs) and its orthologs in A. fumigatus (Table 7). Most of the peptidases shared higher identity to the $A$. fumigatuscp 1 and $c p 2$ genes, except the gene UM226_5845 shared highest identity to TruSCPB (60\% identity, AAS76666). In addition, a gene each from UM 880 (UM880_11056) and UM 226 (UM226_4504) shared 67.67\% and 67.11\% identity, respectively to T. rubrum SCPC [GenBank: AAS76668]. UM 880 and UM 226 consist of four and two putative genes, respectively with $>40 \%$ identity to dermatophytes subtilases and the ortholog of A. fumigatus ALP2. Of these, three UM880 and two UM 226 genes were shown similar to Microsporum canis subtilisin 3 (McaSub3). McaSub3 is required for adherence of the fungus to the epidermal layer of host [70] Although both fungi do not contain the full set of genes encoding subtilases, the potential of their keratinolytic activity shall not be neglected as it is suggested that the multiple keratinases work in concert to ensure successful pathogenesis [71].

Apart from causing superficial skin and nails infections, N. dimidiatum was reported to cause invasive infections [72,73]. In UM 880, six secreted metallopeptidases similar to the Pleurotus ostreatus peptidase PoFE (M43.008) were identified. The conserved active residue and metal ligands were identified (Fig S7). The PoFE carries fibrinolytic activity and was shown able to dissolve blood clots by degrading the fibrin polymers [74]. As the coagulation and deposition of fibrin resulted from inflammation are essential host defense mechanism against infectious agents [75], the fibrinolysis reaction by these peptidases might enable the invasion of the fungus in human.

\section{Antifungal susceptibility profile and drug resistance genes}

Given that the lack of established guidelines on MIC breakpoints for dematiaceous fungi, MIC of $\leq 1 \mu \mathrm{g} / \mathrm{mL}$ was used as an indicator of potential susceptibility for the tested drugs as previously noted [24]. Neoscytalidium dimidiatum UM 880 and B. papendorfii UM 226 were susceptible to posaconazole, voriconazole, fluconazole, ketoconazole, anidulafungin, caspofungin, and amphotericin B. Neoscytalidium dimidiatum UM 880 was resistant to itraconazole ( $\mathrm{MIC}=4 \mu \mathrm{g} / \mathrm{mL})$, whereas $B$. papendorfii UM 226 was susceptible to itraconazole $(\mathrm{MIC}=0.012 \mu \mathrm{g} / \mathrm{mL})(\mathrm{Table} 8)$. The molecular mechanisms of azole resistance have been well described in yeast and mold isolates. Potential resistance mechanisms include alterations in the target enzyme (lanosterol 14a demethylase), or overexpression of various efflux pumps [76,77,78]. Lanosterol 14a demethylase is encoded by the ERG11/CYP51 gene, is targeted by azoles drugs to inhibit fungal ergosterol biosynthesis. A putative Cyp51A (UM880_6807, 69\% identical to Aspergillus flavus) and ERG11 (UM880_1345, 66\% identical to Leptosphaeria maculans) were identified in N. dimidiatum UM 880; a putative ERG11 (UM226_4997, 88\% identical to Leptosphaeria maculans) were identified in B. papendorfii UM 226. These three protein sequences were compared to the previously published ERG11 protein sequences from azole-susceptible Candida albicans isolates (GenBank accession no. XM_711668 and AIX03623). Common ERG11 mutations (A114S, Y132F, K143R, F145L, S405F, D446E, G448E, G450E, and G464S) that associated with azole resistance [79,80] were not observed in UM880_6807, UM880_1345, and UM226_4997. A nonsynonymous CYP51A mutation (M172V) that observed in Aspergillus fumigatus [78] were identified in N. dimidiatum UM880_6807 and UM880_1345 but not in B. papendorfii UM226_4997 (Fig S8). Furthermore, the UM880_6807 and UM880_1345 contain a mutation at the codon 255 (D255K) which was not identified in UM226_4997 as well as in azole-susceptible $C$. albicans [79,80] and $A$. fumigatus [78].

Page $9 / 32$ 
High level expression of major facilitator superfamily (MFS) transporter encoded by MDR1 (multidrug resistance) and ATP-binding cassette (ABC) transporters encoded by CDR1 and CDR2 (Candida drug resistance) are responsible for azoles resistance development [81,82]. Neoscytalidium dimidiatum UM 880 harbours three MDR1 (UM880_2808, UM880_8079, and UM880_9846) and a CDR1 (UM880_4210). As previously noted [83,84], UM880_2808, UM880_8079, and UM880_9846 were predicted to contain 12 putative transmembrane domains by TMpred (Fig S9), suggesting they harbour transmembrane transporter activity. The Ndt80p transcription factor plays a key role to modulate azole tolerance by regulating ERG11 gene expression [84]. The binding of Ndt80p to the promoters of ERG biosynthetic genes is essential to activate their transcription in response to azole drugs [85]. Neoscytalidium dimidiatum UM 880 contains a putative Ndt80 transcription factor (UM880_7612, 87\% identical to Neofusicoccum parvum UCRNP2). The 500 bp upstream regions of the UM880_6807, UM880_1345, and UM880_4210 genes were analyzed in silico. The putative promoter sequence was analyzed for potential NDT80 transcription factor binding sites using JASPAR core fungi database [86]. Among them, only UM880_1345 gene contains a putative Ndt80 binding consensus located between -70 and -90 (data not shown). Neither the MDR1, CDR1 nor NDT80 transcription factor was not found in the B. papendorfii UM 226 genome.

Apart from ABC transporters CDR1 and CDR2, CDR4 has been reported to be involved in azole resistance in filamentous fungi [87]. Knockout of the CDR4 gene increased the susceptibility of Neurospora crassa to azole drugs, including ketoconazole, fluconazole, and itraconazole [87]. We identified a putative CDR4 (UM880_8630, 85\% identical to N. parvum UCRNP2) in N. dimidiatum UM 880 but not in B. papendorfii UM 226 genome. Taken together, the ERG11, MDR1, CDR1, CDR4, and the NDT80 transcription factor may function in concert to modulate ergosterol metabolism to confer low itraconazole resistance in the $N$. dimidiatum UM 880.

\section{Comparative nutrient profiling of $N$. dimidiatum UM 880 and B. papendorfii UM 226}

Phenotype microarrays (PM) analysis was used to obtain a global understanding of the nutritional metabolism of $N$. dimidiatum UM 880 and $B$. papendorfii UM 226. A total of 379 different compounds were tested using PM plates 1 to 8, including 190 different carbon sources, 95 nitrogen sources, 59 phosphorus sources, 35 sulfur sources, 94 nutrient supplements, and 282 peptide nitrogen sources. Phenotypic differences based on their ability to metabolize carbon, nitrogen, phosphorous, sulfur, nutrient supplements, as well as peptide nitrogen sources were noted.

By KEGG annotation analysis, the genes predicted to be involved in central metabolic pathways for carbohydrates utilisation were more abundant in UM 226 compared to UM 880 (Fig 3B). In agreement with the KEGG data (Fig 3B), UM 226 (62 carbon sources) was metabolically active on carbon source as compared to UM 880 (19 carbon sources) (Table 9). PM analysis showed that UM 226 is capable of using simple sugars and complex carbohydrates as carbon sources for growth. UM 226 can utilise monosaccharides (a-D-glucose, D-fructose, D-Psicose, D-ribose, L-rhamnose, Dxylose), disaccharides (maltose, sucrose, D-trehalose), trisaccharide (raffinose), tetrasaccharide (stachyose), synthetic sugar (lactulose), as well as polysaccharides (mannan, pectin). In addition, UM 226 could utilise a wide range of sugar alcohol (adonitol, dulcitol, i-Erythritol, D-arabitol, Dmannitol, maltitol, xylitol) and carboxylic acids (D-tartaric acid, $\delta$-amino valeric acid, $\gamma$-hydroxybutyric acid, $\beta$-hydroxybutyric acid, a-keto valeric acid, $Y$-amino butyric acid), which could not utilised by UM 880. Based on KEGG analysis, genes involved in glycolysis, gluconeogenesis, pentose phosphate pathway, fructose, mannose, sucrose, galactose, and starch metabolism were annotated in both UM 880 and UM 226 although no evidence from PM analysis to support UM 880 activities on these carbon substrates (Table S11). Overall, UM 226 has the greater ability to utilise a wide array of carbon substrates, a reflection of its broad carbohydrate utilisation.

PM data revealed markedly differences in nitrogen metabolism when UM 880 and UM 226 were compared (Table 9) although a comparable number of genes predicted for nitrogen metabolism was annotated in UM 880 and UM 226 genomes (29 and 31 genes, respectively) (Table S12). UM 880 was able to utilize 56 out of 95 nitrogen sources, while UM 226 could utilise only on eight of the tested nitrogen substrates. The nitrogen sources selective for UM 226 growth were organic nitrogen (ammonia), uric acid, L-amino acid (L-proline, L-alanine, and L-glutamine), D-amino acid (Dvaline), and dipeptides (Ala-Asp and Gly-GIn). Interestingly, we found that UM 880 genome contains a nitrate transport protein (UM880_1228) and a nitrite reductase protein (UM880_5592), which are found to be absent in UM 226. Furthermore, it should be noted that no phosphorus, sulfur, and nutritional supplements was utilised by UM 226 (Table 9). On the other hand, UM 880 was found able to utilise 14 phosphorus, sulfur, as well as a nutritional supplement. In addition, UM 880 showed the greater ability to metabolize peptide nitrogen substrates (183 peptide nitrogen substrates) as compared to the UM 226 (117 peptide nitrogen substrates). Taken together, we speculated that the reduced utilisation of carbon sources could be compensated by the utilisation of nitrogen, phosphorus, sulfur, nutritional supplement, and peptide nitrogen. Although $N$. dimidiatum UM 880 and $B$. papendorfii UM 226 contain a comparable number and distribution of CAZymes, their nutrient utilisation profile varies significantly. PM data revealed that UM 880 could utilise a diverse array of substrates, which further demonstrate its metabolic versatility. Thus, it is not surprising that $N$. dimidiatum has a broad geographical and host range [7]. In contrast, UM 226 utilizes limited sources of nutrients as compared to that of UM 880.

\section{Comparative chemical sensitivity profile of $N$. dimidiatum UM 880 and $B$. papendorfii UM 226}

Fungi are commonly exposed to broadly fluctuating environmental conditions where adaptation is crucial for their survival. In particular, an ability to respond to a wide $\mathrm{pH}$ range allows them to cope with rapid changes in their extracellular settings. In fact, $\mathrm{pH}$ is also one of the major environmental factors affecting pathogenicity. Therefore, the growth of B. papendorfii UM 226 and $N$. dimidiatum UM 880 at various pH were analyzed in this study.

Page $10 / 32$ 
Our results revealed that UM 226 grew better compared to UM 880 at all pH range. UM226 grew well at a wide range of pH ranging from strongly acidic to alkaline ( $\mathrm{pH} 3.5$ to 10), with an optimum growth between $\mathrm{pH} 4$ and 8. On the other hand, the maximum growth of UM 880 was at $\mathrm{pH} 4.5-5.5$. Lambers et al. (2006) has demonstrated that the $\mathrm{pH}$ of natural skin surface is at an average of 4.7 [88]. The ability of both UM 226 and UM 880 to grow at this $\mathrm{pH}(\sim \mathrm{pH} 4.7)$ may account for their high occurrence in dermal surface and hence causing dermatitis disorder. In addition, these fungal especially UM 226 can most likely continue to grow on the skin surface even after application of cosmetic product or soap since they can adapt to adverse alkaline $\mathrm{pH}$ condition (Fig 6).

Noting the versatility of these strains at wide $\mathrm{pH}$ range, we also evaluated the efficiency of various supplementation on their potential inhibitory effect at both acidic and alkaline conditions. Our results indicated that the supplementation of nitrogen sources did not affect the growth of UM 880 at alkaline condition (Fig 7). Interestingly, supplementation with L-histidine, L-phenylalanine and L-tryptophan are shown to exhibit additional inhibitory effect for UM 226 at alkaline condition ( $\mathrm{pH}$ 9.5). On the other hand, supplementation of anthranilic acid is shown to inhibit the growth of UM 226 and UM 880 at pH 4.5 (a pH close to natural skin surface condition) as their growth in media supplemented with anthranilic acid were significantly $(\mathrm{P}<0.05)$ lower $(60-92 \%$ lower) compared to the control (Fig 8). Other nitrogenous sources evaluated in this study had very limited effect on the growth of both strains. This may suggest that supplementation of anthranilic acid L-histidine and L-tryptophan on cosmetic agents such as alkaline soap or topical creams may likely inhibit the growth of UM 226 and UM 880 on skin surface, hence potentially reducing the risk for dermatitis disease due to these fungi.

The growth of UM 226 in saline condition was concentration dependent and generally showed a better salt tolerance as compared to UM 880 (Fig 9 ). Although there was a gradual decreased in the growth of UM 226 as the concentration of $\mathrm{NaCl}$ increases, the mycelial growth of UM 226 remained high (above $\mathrm{OD}=1$ ) even at $9 \% \mathrm{NaCl}$. Unlike UM 226, low concentration of $\mathrm{NaCl}(2-3 \% \mathrm{NaCl})$ promoted the growth of UM 880 and the growth start to decline as the concentration exceed $4 \% \mathrm{NaCl}$. In order to increase the ability to grow at high salinity condition, microorganism would require a special adaptation to maintain the osmotic equilibrium across the cell membrane. Cells of almost all organisms commonly utilised organic-osmolytes mechanism when exposed to hyperosmolality, high salt or solute condition [88]. Therefore, we also screened for the potential osmolytes by including a range of compounds in the solution containing $6 \% \mathrm{NaCl}$. Unlike most microorganism which required betaine or glycerol to restore their osmotolerance properties [89], our data showed that both glutamic acid and proline provided a better osmoprotectant effect for both strains of fungal especially UM 226 as compared to betaine (Fig 10). On the other hand, dimethyl sulphonyl propionate was shown to exert additional inhibitory effect for both strains when cultured in $6 \% \mathrm{NaCl}$ condition (Fig 10). This data would be important to provide a fundamental knowledge in developing potential drug targets for antifungal strategies for both these strains.

In addition to $\mathrm{NaCl}$, this study also evaluated the effect of various other salt including sodium lactate, sodium formate, sodium nitrate and sodium nitrite. UM 226 and UM 880 showed a concentration dependent growth in all the salt media (Table S13). Similar observation was demonstrated in media containing urea where a gradual decreased of mycelial was observed in both strain as the concentration of urea increases. It is critical to understand the growth pattern of the fungi in urea because urea has been commonly used in treatment of dermatological diseases and as potent dermal emollient [90]. In general, UM 226 was shown to exhibit better $(p<0.05)$ mycelial growth compared to UM 880 in media containing urea. Our results suggest that increasing the concentration of urea may decrease the mycelial growth. The mycelial growth for both strains drastically decreased by $88-91 \%$ in media containing $7 \%$ urea compared to that of containing $2 \%$. The $\mathrm{OD}_{750}$ for both strains were almost negligible in media containing 7\% urea (Fig 11). Previous study has demonstrated that Neoscytalidium infections, even always superficial, are often difficult to treat [91] while information for treatment of $B$. papendorfii in skin is remained limited. Therefore, our data is important to provide a fundamental information for clinical management of these fungi.

\section{Conclusion}

In this study, we take an organized approach to expand our knowledge of the genomic, genetic, as well as metabolic profiles of these plant pathogens that were isolated from humans. Comparative genomics of $N$. dimidiatum and $B$. papendorfii revealed several striking differences in genome size, GC content, gene content, and transposons. The genome sequences further demonstrated a remarkable difference in the amount and types of putative secondary metabolites. Unlike N. dimidiatum, B. papendorfii contain a gene encoding dimethylallyl tryptophan synthase (DMATS). The putative 6-methylsalicylic-acid synthase gene was unique to $N$. dimidiatum. The biological function of most of this predicted gene remains to be determined to gain insight whether it contribute to the biosynthesize of various antibiotics. A survey of the CAZYmes in the $N$. dimidiatum and $B$. papendorfii with other fungi from different lifestyles showed that they have similar mode of infection in plants. The distribution of CAZyme-encoding genes demonstrated a preference of $N$. dimidiatum and $B$. papendorfii for hemicellulose and pectin as nutrient sources. Additionally, rare human diseases caused by these fungi may be associated with the presence of several putative keratinolytic peptidases. In particular, we also identified several genes associated with azole resistance of $N$. dimidiatum, which were missed in the $B$. papendorfii genome. Our PM analysis proved that $N$. dimidiatum could utilise a diverse array of nutrient sources particularly noticeable in the case of nitrogen, phosphorus and sulfur sources as compared to $B$. papendorfii that mainly utilizes carbon as nutrient source. The most significant finding in the data was that $B$. papendorfii is a salttolerant mold, which can adapt to the wide $\mathrm{pH}$ range, suggesting it can tolerate and survive in hostile and diverse environments. However, substrate utilisation observed under different growth conditions is still unknown. Therefore, future study on the substrate utilisation under diverse growth conditions is vital to gain better awareness of their lifestyles and subsequently their infection model. 
Declarations

\section{Ethics approval and consent to participate}

Not applicable.

\section{Consent for publication}

Not applicable.

\section{Competing interest}

The authors declared that there is no competing interest.

\section{Funding}

This study was supported by High Impact Research MOE Grant (UM.C/625/1/HIR/MOHE/MED/31, account no: H-20001-00-E000070) from the Ministry of Education Malaysia and Putra Graduate Initiative Grant from Universiti Putra Malaysia (GP-IPS/2017/9526300).

\section{Availability of data and materials}

The datasets supporting the conclusions of this article are included within the article and its additional files.

\section{Author contributions}

Chee Sian Kuan: Conceptualisation; Data curation; Investigation; Methodology; Project administration; Supervision; Validation; Visualisation; Writing - original draft. Kee Peng Ng: Conceptualisation; Supervision; Funding acquisition; Resources; Writing - review \& editing. Su Mei Yew: Data curation; Investigation; Methodology; Writing - review \& editing. Hadiza Umar Meleh: Investigation; Writing - review \& editing. Heng Fong Seow: Writing review \& editing. Kang Nien How: Writing - review \& editing. Siok Koon Yeo: Investigation; Writing - original draft. Jap Meng Jee: Investigation; Methodology. Yung-Chie Tan: Data curation; Software. Wai-Yan Yee: Data curation; Software. Chee-Choong Hoh: Data curation; Software. Rukumani Devi Velayuthan: Investigation; Methodology. Shiang Ling Na: Methodology. Siti Norbaya Masri: Writing - review \& editing. Shu Yih Chew: Data curation; Methodology; Writing - review \& editing. Leslie Thian Lung Than: Conceptualisation; Supervision; Funding acquisition; Resources; Writing review \& editing.

\section{References}

1. Tan DH, Sigler L, Gibas CF, Fong IW. Disseminated fungal infection in a renal transplant recipient involving Macrophomina phaseolina and Scytalidium dimidiatum: case report and review of taxonomic changes among medically important members of the Botryosphaeriaceae. Med Mycol. 2008; 46: 285-292.

2. Crous PW, Slippers B, Wingfield MJ, et al. Phylogenetic lineages in the Botryosphaeriaceae. Stud Mycol. 2006; 55: 235-253.

3. Phillips AJ, Alves A, Abdollahzadeh J, et al. The Botryosphaeriaceae: genera and species known from culture. Stud Mycol. 2013; 76 : 51-167.

4. Machado AR, Pinho DB, Dutra DC, Pereira OL. First report of collar and root rot of physic nut (Jatropha curcas) caused by Neoscytalidium dimidiatum in Brazil. Plant Dis. 2012; 96: 1697.

5. Lan GB, He ZF. First report of brown spot disease caused by Neoscytalidium dimidiatum on Hylocereus undatus in Guangdong, Chinese Mainland. Plant Dis. 2012; 96: 1702.

6. Polizzi G, Aiello D, Vitale A. First report of shoot blight, canker, and gummosis caused by Neoscytalidium dimidiatum on Citrus in Italy. Plant Dis. 2009; 93: 1215.

7. Ray JD, Burgess T, Lanoiselet VM. First record of Neoscytalidium dimidiatum and novaehollandiae on Mangifera indica and N. dimidiatum on Ficus carica in Australia. Australas Plant Dis Notes. 2010; 5: 48-50.

8. Abo Rehab ME, Rashed MF, Ammar MI, El-Morsy SA. Dieback and sooty canker of Ficus trees in Egypt and its control. Pak J Biol Sci. 2014; 17: 364-371.

9. Hassan WA, Pasha AA, Mohammad MB. Sooty canker on some thin bark trees caused by Nattrassia mangiferae. Egypt J Agric Res. 2009; 87: 443-456. 
10. Sadowsky A, Solel Z, Sztejnberg A. Effect of heat-stress predisposition on the development of Scytalidium wilt of 'Star Ruby' grapefruit, caused by Scytalidium lignicola. Eur J Plant Pathol. 2007; 117: 123-127.

11. Hassan WA, Haleem RA, Hassan PH. Effect of heat-stress predisposition on the development of sooty canker caused by Neoscytalidium dimidiatum (Penz.) Crous and Slippers. Acta Agrobot. 2011; 64: 207-212.

12. Elewski BE. Onychomycosis caused by Scytalidium dimidiatum. J Am Acad Dermatol. 1996; 35: 336-338.

13. Lacaz CS, Pereira AD, Heins-Vaccari EM, et al. Onychomycosis caused by Scytalidium dimidiatum. Report of two cases. Review of the taxonomy of the synanamorph and anamorph forms of this coelomycete. Rev Inst Med Trop Sao Paulo. 1999; 41: 319-323.

14. Arrese JE, Pierard-Franchimont C, Pierard GE. Scytalidium dimidiatum melanonychia and scaly plantar skin in four patients from the Maghreb: imported disease or outbreak in a Belgian mosque? Dermatology. 2001; 202: 183-185.

15. Hay RJ. Scytalidium infections. Curr Opin Infect Dis. 2002; 15: 99-100.

16. Rockett MS, Gentile SC, Zygmunt KH, Gudas CJ. Subcutaneous phaeohypomycosis caused by Scytalidium dimidiatum in the foot of an immunosuppressed host. J Foot Ankle Surg. 1996; 35: 350-354.

17. Drouhet E, Dupont B. Laboratory and clinical assessment of ketoconazole in deep-seated mycoses. Am J Med. $1983 ; 74: 30-47$.

18. Geramishoar M, Zomorodian K, Zaini F, et al. First case of cerebral phaeohyphomycosis caused by Nattrassia mangiferae in Iran. Jpn J Infect Dis. $2004 ; 57: 285-286$.

19. Dunn JJ, Wolfe MJ, Trachtenberg J, Kriesel, JD, Orlandi RR, Carroll KC. Invasive fungal sinusitis caused by Scytalidium dimidiatum in a lung transplant recipient. J Clin Microbiol. 2003; 41: 5817-5819.

20. Al-Rajhi AA, Awad AH, Al-Hedaithy SS, Forster RK, Caldwell KC. Scytalidium dimidiatum fungal endophthalmitis. Br J Ophthalmol. 1993; 77: 388390.

21. Li PP, Cao ZY. First report of Bipolaris papendorfii causing corn leaf spot in China. Plant Dis. 2013; $97: 1506$.

22. Valencia-Botín AJ, Kokubu H, Ruiz DR. A brief overview on pitahaya (Hylocereus) diseases. Australas Plant Pathol. 2013; 15: 437-440.

23. Masratul HM, Baharuddin S, Latiffah Z. Identification and molecular characterizations of Neoscytalidium dimidiatum causing stem canker of red-fleshed dragon fruit (Hylocereus polyrhizus) in Malaysia. J Phytopathol. 2013; 161: 841-849.

24. Yew SM, Chan CL, Lee KW, et al. A five-year survey of dematiaceous fungi in a tropical hospital reveals potential opportunistic species. PLoS One. 2014; 9: e104352.

25. Kuan CS, Yew SM, Toh YF, et al. Dissecting the fungal biology of Bipolaris papendorfii: from phylogenetic to comparative genomic analysis. DNA Res. 2015; 22: 219-232.

26. Zerbino DR, Birney E. Velvet: algorithms for de novo short read assembly using de Bruijn graphs. Genome Res. 2008 ; $18: 821-829$.

27. Boetzer M, Henkel CV, Jansen HJ, Butler D, Pirovano W. Scaffolding pre-assembled contigs using SSPACE. Bioinformatics. 2011 ; 27 : 578-579.

28. Boetzer M, Pirovano W. Toward almost closed genomes with GapFiller. Genome Biol. 2012; 13 : R56.

29. Nadalin F, Vezzi F, Policriti A. GapFiller: a de novo assembly approach to fill the gap within paired reads. BMC Bioinformatics. $2012 ; 13$ : S8.

30. Lagesen K, Hallin P, Rodland EA, Staerfeldt HH, Rognes T, Ussery DW. RNAmmer: consistent and rapid annotation of ribosomal RNA genes. Nucleic Acids Res. 2007; 35: 3100-3108.

31. Lowe TM, Eddy SR. tRNAscan-SE: a program for improved detection of transfer RNA genes in genomic sequence. Nucleic Acids Res. 1997; 25: 955-964.

32. Lomsadze A, Ter-Hovhannisyan V, Chernoff YO, Borodovsky M. Gene identification in novel eukaryotic genomes by self-training algorithm. Nucleic Acids Res. 2005; 33: 6494-6506.

33. Conesa A, Gotz S, Garcia-Gomez JM, Terol J, Talon M, Robles M. Blast2GO: a universal tool for annotation, visualization and analysis in functional genomics research. Bioinformatics. 2005; 21: 3674-3676.

34. Tatusov RL, Fedorova ND, Jackson JD, et al. The COG database: an updated version includes eukaryotes. BMC Bioinformatics. $2003 ; 4: 41$.

35. Quevillon E, Silventoinen V, Pillai S, et al. InterProScan: protein domains identifier. Nucleic Acids Res. 2005; 33: W116-120.

36. Ohm RA, Feau N, Henrissat B, et al. Diverse lifestyles and strategies of plant pathogenesis encoded in the genomes of eighteen Dothideomycetes fungi. PLoS Pathog. 2012; 8: e1003037.

37. Zhao Z, Liu H, Wang C, Xu JR. Correction: comparative analysis of fungal genomes reveals different plant cell wall degrading capacity in fungi. BMC Genomics. 2014; 15: 6.

38. Rawlings ND, Barrett AJ, Bateman A. MEROPS: the database of proteolytic enzymes, their substrates and inhibitors. Nucleic Acids Res. 2012; 40: D343-350.

39. Petersen TN, Brunak S, von Heijne G, Nielsen H. SignalP 4.0: discriminating signal peptides from transmembrane regions. Nat Methods. 2011; 8: 785-786. 
40. Krogh A, Larsson B, von Heijne G, Sonnhammer EL. Predicting transmembrane protein topology with a hidden Markov model: application to complete genomes. J Mol Biol. 2001; 305: 567-580.

41. Khaldi N, Seifuddin FT, Turner G, et al. SMURF: Genomic mapping of fungal secondary metabolite clusters. Fungal Genet Biol. 2010; 47: 736741.

42. Sigler L, Summerbell RC, Poole L, et al. Invasive Nattrassia mangiferae infections: case report, literature review, and therapeutic and taxonomic appraisal. J Clin Microbiol. 1997; 35: 433-440.

43. Daboussi MJ. Fungal transposable elements and genome evolution. Genetica. 1997; 100: 253-260.

44. Teixeira MM, de Almeida LG, Kubitschek-Barreira P, et al. Comparative genomics of the major fungal agents of human and animal Sporotrichosis: Sporothrix schenckii and Sporothrix brasiliensis. BMC Genomics. 2014; 15: 943.

45. Santana MF, Silva JC, Mizubuti ES, et al. Characterization and potential evolutionary impact of transposable elements in the genome of Cochliobolus heterostrophus. BMC Genomics. 2014; 15: 536.

46. Brillet B, Bigot Y, Auge-Gouillou C. Assembly of the Tc1 and mariner transposition initiation complexes depends on the origins of their transposase DNA binding domains. Genetica. 2007; 130: 105-120.

47. Galperin MY, Koonin EV. 'Conserved hypothetical' proteins: prioritization of targets for experimental study. Nucleic Acids Res. 2004; 32: 54525463.

48. Leach MD, Brown AJ. Posttranslational modifications of proteins in the pathobiology of medically relevant fungi. Eukaryot Cell. 2012; 11: 98108.

49. Bushley KE, Turgeon BG. Phylogenomics reveals subfamilies of fungal nonribosomal peptide synthetases and their evolutionary relationships. BMC Evol Biol. 2010; 10: 26.

50. Kroken S, Glass NL, Taylor JW, Yoder, OC, Turgeon BG. Phylogenomic analysis of type I polyketide synthase genes in pathogenic and saprobic ascomycetes. Proc Natl Acad Sci USA. 2003; 100: 15670-15675.

51. Yang G, Rose MS, Turgeon BG, Yoder OC. A polyketide synthase is required for fungal virulence and production of the polyketide T-toxin. Plant Cell. 1996; 8: 2139-2150.

52. Panaccione DG, Scott-Craig JS, Pocard JA, Walton JD. A cyclic peptide synthetase gene required for pathogenicity of the fungus Cochliobolus carbonum on maize. Proc Natl Acad Sci USA. 1992; 89: 6590-6594.

53. Jahn B, Boukhallouk F, Lotz J, Langfelder K, Wanner G, Brakhage AA. Interaction of human phagocytes with pigmentless Aspergillus Infect Immun. 2000; 68: 3736-3739.

54. Eliahu N, Igbaria A, Rose MS, Horwitz BA, Lev S. Melanin biosynthesis in the maize pathogen Cochliobolus heterostrophus depends on two mitogen-activated protein kinases, Chk1 and Mps1, and the transcription factor Cmr1. Eukaryot Cell. 2007; 6: $421-429$.

55. Wang Y, Wang J, Cheong YH, Hur JS. Three new non-reducing polyketide synthase genes from the lichen-forming fungus Usnea longissima. Mycobiology. 2014; 42: 34-40.

56. Moriwaki A, Kihara J, Kobayashi T, Tokunaga T, Arase S, Honda Y. Insertional mutagenesis and characterization of a polyketide synthase gene (PKS1) required for melanin biosynthesis in Bipolaris oryzae. FEMS Microbiol Lett. 2004; 238: 1-8.

57. Ding W, Lei C, He Q, Zhang Q, Bi Y, Liu W. Insights into bacterial 6-methylsalicylic acid synthase and its engineering to orsellinic acid synthase for spirotetronate generation. Chem Biol. 2010; 17: 495-503.

58. Weinberg ED. The role of iron in protozoan and fungal infectious diseases. J Eukaryot Microbiol. 1999; 46: $231-238$.

59. Schrettl M, Bignell E, Kragl C, et al. Siderophore biosynthesis but not reductive iron assimilation is essential for Aspergillus fumigatus J Exp Med. 2004; 200: 1213-1219.

60. Oide S, Moeder W, Krasnoff S, et al. NPS6, encoding a nonribosomal peptide synthetase involved in siderophore-mediated iron metabolism, is a conserved virulence determinant of plant pathogenic ascomycetes. Plant Cell. 2006; 18: 2836-2853.

61. Condon BJ, Oide S, Gibson DM, Krasnoff SB, Turgeon BG. Reductive iron assimilation and intracellular siderophores assist extracellular siderophore-driven iron homeostasis and virulence. Mol Plant Microbe Interact. 2014; 27: 793-808.

62. Haas H, Eisendle M, Turgeon BG. Siderophores in fungal physiology and virulence. Annu Rev Phytopathol. 2008 ; 46: $149-187$.

63. Condon BJ, Wu D, Kraševec N, Horwitz BA, Turgeon BG. Comparative genomics of Cochliobolus phytopathogens, In Genomics of PlantAssociated Fungi: Monocot Pathogens. Springer-Verlag Berlin Heidelberg; 2004: 41-67.

64. Lian T, Simmer MI, D'Souza CA, et al. Iron-regulated transcription and capsule formation in the fungal pathogen Cryptococcus neoformans. Mol Microbiol. 2005; 55: 1452-1472.

65. Eichhorn H, Lessing F, Winterberg B, et al. A ferroxidation/permeation iron uptake system is required for virulence in Ustilago maydis. Plant Cell. 2006; 18: 3332-3345.

66. Ospina-Giraldo MD, Griffith JG, Laird EW, Mingora C. The CAZyome of Phytophthora : a comprehensive analysis of the gene complement coding for carbohydrate-active enzymes in species of the genus Phytophthora. BMC Genomics. 2010; 11: 525. 
67. Langston JA, Shaghasi T, Abbate E, Xu F, Vlasenko E, Sweeney MD. Oxidoreductive cellulose depolymerization by the enzymes cellobiose dehydrogenase and glycoside hydrolase 61. Appl Environ Microbiol. 2011; 77: 7007-7015.

68. Burmester A, Shelest E, Glockner G, et al. Comparative and functional genomics provide insights into the pathogenicity of dermatophytic fungi. Genome Biol. 2011; 12: R7.

69. Monod M, Lechenne B, Jousson O, et al. Aminopeptidases and dipeptidyl-peptidases secreted by the dermatophyte Trichophyton rubrum. Microbiology. 2005; 151: 145-155.

70. Bagut ET, Baldo A, Mathy A, et al. Subtilisin Sub3 is involved in adherence of Microsporum canis to human and animal epidermis. Vet Microbiol. 2012; 160: 413-419.

71. Shi Y, Niu Q, Yu X, et al. Assessment of the function of SUB6 in the pathogenic dermatophyte Trichophyton mentagrophytes. Med Mycol. 2016; 54: 59-71.

72. Elinav H, Izhar U, Benenson S, et al. Invasive Scytalidium dimidiatum infection in an immunocompetent adult. J Clin Microbiol. 2009; 47: 12591263.

73. Garinet S, Tourret J, Barete S, et al. Invasive cutaneous Neoscytalidium infections in renal transplant recipients: a series of five cases. BMC Infect Dis 2015; 15: 535 .

74. Shen MH, Kim JS, Sapkota K, et al. Purification, characterization, and cloning of fibrinolytic metalloprotease from Pleurotus ostreatus J Microbiol Biotechnol. 2007; 17: 1271-1283.

75. Bergmann S, Hammerschmidt S. Fibrinolysis and host response in bacterial infections. Thromb Haemost. 2007; 98: 512-520.

76. White TC. Increased mRNA levels of ERG16, CDR, and MDR1 correlate with increases in azole resistance in Candida albicans isolates from a patient infected with human immunodeficiency virus. Antimicrob Agents Chemother. 1997; 41: 1482-1487.

77. Perea S. [Azole resistance in Candida albicans]. Rev Esp Quimioter. 2000; 13: 314-317.

78. Howard SJ, Cerar D, Anderson MJ, et al. Frequency and evolution of azole resistance in Aspergillus fumigatus associated with treatment failure. Emerg Infect Dis. 2009; 15: 1068-1076.

79. Flowers SA, Colon B, Whaley SG, Schuler MA, Rogers PD. Contribution of clinically derived mutations in ERG11 to azole resistance in Candida albicans. Antimicrob Agents Chemother. 2015; 59: 450-460.

80. Xiang MJ, Liu JY, Ni PH, et al. Erg11 mutations associated with azole resistance in clinical isolates of Candida albicans. FEMS Yeast Res. 2013; 13: 386-393.

81. Tsao S, Rahkhoodaee F, Raymond M. Relative contributions of the Candida albicans ABC transporters Cdr1p and Cdr2p to clinical azole resistance. Antimicrob Agents Chemother. 2009; 53: 1344-1352.

82. Anderson JB. Evolution of antifungal-drug resistance: mechanisms and pathogen fitness. Nat Rev Microbiol. $2005 ; 3: 547-556$.

83. Law CJ, Maloney PC, Wang DN. Ins and outs of major facilitator superfamily antiporters. Annu Rev Microbiol. 2008; 62: $289-305$.

84. Pao SS, Paulsen IT, Saier MH Jr. Major facilitator superfamily. Microbiol Mol Biol Rev. 1998; 62: 1-34.

85. Sellam A, Tebbji F, Nantel A. Role of Ndt80p in sterol metabolism regulation and azole resistance in Candida albicans. Eukaryot Cell. 2009; 8: 1174-1183.

86. Mathelier A, Zhao X, Zhang AW, et al. JASPAR 2014: an extensively expanded and updated open-access database of transcription factor binding profiles. Nucleic Acids Res. 2014; 42: D142-147.

87. Zhang Y, Zhang Z, Zhang X, et al. CDR4 is the major contributor to azole resistance among four Pdr5p-like ABC transporters in Neurospora crassa. Fungal Biol. 2012; 116: 848-854.

88. Lambers H, Piessens S, Bloem A, Pronk H, Finkel P. Natural skin surface pH is on average below 5 , which is beneficial for its resident flora. Int $\mathrm{J}$ Cosmet Sci 2006; 28: 359-370.

89. Burg MB, Ferraris JD. Intracellular organic osmolytes: function and regulation. J Biol Chem. 2008; 283: 7309-7313.

90. Pan M, Heinecke G, Bernardo S, Tsui C, Levitt J. Urea: a comprehensive review of the clinical literature. Dermatol Online J. $2013 ; 19: 20392$.

91. Khan ZU, Ahmad S, Joseph L, Chandy R. Cutaneous phaeohyphomycosis due to Neoscytalidium dimidiatum: first case report from Kuwait. J Mycol Med. 2009; 19: 138-42.

\section{Tables}


Table 1

Genome features of N. dimidiatum UM 880 and B. papendorfii UM 226

\begin{tabular}{|lll|}
\hline Details & N. dimidiatum UM 880 & B. papendorfii UM 226 \\
\hline Sequencing depth & $\sim 93 \times$ & $\sim 87 \times$ \\
\hline Assembly Size (bp) & $42,687,746$ & $33,397,470$ \\
\hline Number of contigs ( $\geq 200$ bp) & 2,203 & 374 \\
\hline Total contigs size (bp) & $42,571,090$ & - \\
\hline Contigs N50 (bp) & 35,797 & 146,099 \\
\hline Number of scaffolds $(\geq 1000$ bp) & 110 & - \\
\hline Total scaffolds size (bp) & $42,687,746$ & - \\
\hline Scaffolds N50 (bp) & $1,952,936$ & - \\
\hline G + C content (\%) & 54.16 & 50.65 \\
\hline
\end{tabular}

Table 2

Transposable element composition in the N. dimidiatum UM 880 and B. papendorfii UM 226 genomes

\begin{tabular}{|llllll|}
\hline \multirow{2}{*}{ Class } & Family & \multicolumn{2}{l}{ N. dimidiatum UM 880} & \multicolumn{2}{l|}{ B. papendorfii UM 226} \\
\cline { 2 - 6 } & & Copies & Percentage (\%) & Copies & Percentage (\%) \\
\hline I & DDE_1 & 23 & 0.030 & 422 & 1.11 \\
\hline Gypsy & 83 & 0.192 & 279 & 0.67 \\
\hline LINE & 88 & 0.186 & 54 & 0.24 \\
\hline TY1_Copia & 34 & 0.065 & 87 & 0.29 \\
\hline Itr_Roo & 0 & 0 & 2 & 0.00001 \\
\hline helitronORF & 5 & 0.009 & 4 & 0.01 \\
\hline hAT & 3 & 0.006 & 21 & 0.05 \\
\hline mariner & 3 & 0.008 & 55 & 0.11 \\
\hline mariner_ant1 & 1 & 0.002 & 6 & 0.01 \\
\hline MuDR_A_B & 11 & 0.012 & 19 & 0.01 \\
\hline cacta & 9 & 0.003 & 10 & 0.00003 \\
\hline Crypton & 1 & 0.001 & 0 & 0 \\
\hline Total & 261 & 0.51 & 959 & 2.49 \\
\hline
\end{tabular}


Table 3

Gene model of N. dimidiatum UM 880 and B. papendorfii UM 226 genomes.

\begin{tabular}{|llll|}
\hline \multirow{2}{*}{ Gene model } & \multicolumn{2}{l|}{ Fungal species } \\
\cline { 2 - 4 } & N. dimidiatum UM 880 & B. papendorfi UM 226 \\
\hline Number of predicted genes $(>=99 \mathrm{bp})$ & 12,320 & 11,015 \\
\hline Average gene length & 1,451 & 1,425 \\
\hline Average number of exons per gene & & 3.09 & 2.95 \\
\hline Annotated protein coding regions & NCBI nr & 11,577 & 10,708 \\
\cline { 2 - 4 } & SwissProt & 7,656 & 6,963 \\
\cline { 2 - 4 } & InterPro & 9,155 & 8,283 \\
\hline tRNAs & & 137 & 128 \\
\hline rRNAs & & 23 & 18 \\
\hline
\end{tabular}


Table 4

Genes assigned to the "Cellular Component", "Molecular Function”, and "Biological Process" categories of GO in UM 880 and UM226

\section{GO ontology}

Cellular component

1. Cell (GO:0005623)

Membrane (G0:0016020)

Intracellular part (G0:0044424)

2. Membrane-enclosed lumen (G0:0031974)

3. Macromolecular complex (G0:0032991)

Protein complex (G0:0043234)

4. Organelle (GO:0043226)

Molecular function

1. Oxidoreductase activity (G0:0016491)

2. Transferase activity (G0:0016740)

3. Hydrolase activity (G0:0016787)

4. Ligase activity (G0:0016874)

5. Transporter activity (GO:0005215)

Transmembrane transporter activity (G0:0022857)

Substrate-specific transporter activity (GO:0022892)

Vitamin transporter activity (GO:0051183)

Cofactor transporter activity (G0:0051184)

6. Binding (G0:0005488)

Nucleotide binding (G0:0000166)

Nucleoside binding (G0:0001882)

Nucleic acid binding (G0:0003676)

Vitamin binding (G0:0019842)

lon binding (GO:0043167)

Tetrapyrrole binding (G0:0046906)

Cofactor binding (G0:0048037)

7. Electron carrier activity (G0:0009055)

8. Transcription regulator activity (G0:0030528)

Two-component response regulator activity (G0:0000156)

Transcription factor activity (G0:0003700)

9. Translation factor activity, nucleic acid binding (G0:0008135)

\section{Biological process}

1. Metabolic process (GO:0008152)

Nitrogen compound metabolic process (G0:0006807)

Macromolecule metabolic process (G0:0043170)

Primary metabolic process (G0:0044238)

Oxidation reduction (G0:0055114)

\section{Number of genes in UM $880 \quad$ Number of genes in UM $226 \quad p$-value}

\begin{tabular}{lll}
6,001 & 5,801 & 0 \\
\hline 2,604 & 2,412 & 0.002 \\
\hline 5,402 & 5,212 & 0.003 \\
1,217 & 1,332 & 0.007 \\
1,692 & 1,941 & 0 \\
1,294 & 1,541 & 0 \\
4,726 & 4,580 & 0.043
\end{tabular}

$1,243 \quad 1,068$

$9801125 \quad 0$

$\begin{array}{lll}1,452 & 1,566 & 0.011\end{array}$

$223 \quad 264 \quad 0.05$

$\begin{array}{lll}871 & 779 & 0.023\end{array}$

$\begin{array}{lll}755 & 645 & 0.003\end{array}$

$\begin{array}{lll}768 & 680 & 0.021\end{array}$

$49 \quad 28 \quad 0.018$

$\begin{array}{lll}85 & 54 & 0.009\end{array}$

$4,487 \quad 4,788 \quad 0$

$8491,166 \quad 0$

$527 \quad 803 \quad 0$

$839 \quad 1160 \quad 0$

$64111 \quad 0$

$968 \quad 1,306 \quad 0$

$\begin{array}{lll}80 & 128 & 0.001\end{array}$

$325 \quad 431 \quad 0$

$100 \quad 155 \quad 0$

$260382 \quad 0$

6

113

28

0

$210 \quad 0$

69

94

0.044

5,004

2,433

5,139

0.002

2,656

0

2,892

0

4,105

0.002

3,943

771

0 


\begin{tabular}{|lllll|}
\hline \multicolumn{2}{|l}{ GO ontology } & Number of genes in UM 880 & Number of genes in UM 226 & p-value \\
\hline 2. & Pigmentation (GO:0043473) & 1,944 & 2,054 & 0.022 \\
\hline 3. & Response to stimulus (GO:0050896) & 2,145 & 1,981 & 0.006 \\
\hline & Response to chemical stimulus (GO:0042221) & 1,110 & 976 & 0.003 \\
\hline 4. & Regulation of biological process (GO:0050789) & 1,988 & 2,107 & 0.014 \\
\hline
\end{tabular}

Table 5

Numbers of backbone-genes required for the biosynthesis of secondary metabolites in N. dimidiatum UM 880 and B. papendorfii UM 226 genomes

\begin{tabular}{|lll|}
\hline Classifications & \multicolumn{2}{l|}{ Fungal species } \\
\cline { 2 - 3 } & N. dimidiatum UM 880 & B. papendorfii UM 226 \\
\hline PKS & 19 & 17 \\
\hline PKS-like & 2 & 2 \\
\hline NRPS & 8 & 4 \\
\hline NRPS-like & 8 & 7 \\
\hline HYBRID & 2 & 1 \\
\hline DMAT & 0 & 1 \\
\hline Total & 39 & 32 \\
\hline $\begin{array}{l}\text { PKS, polyketide synthetase; NRPS, non-ribosomal peptide synthetase; HYBRID, hybrid PKS-NRPS enzyme; DMAT, dimethylallyl tryptophan } \\
\text { synthase }\end{array}$ & \\
\hline
\end{tabular}

Table 6

Total number of putative keratinolytic peptidases families in UM 880, UM

226, A. benhamiae, and T. verrucosum

\begin{tabular}{|lllll|}
\hline Secreted protein & UM $\mathbf{8 8 0}$ & UM 226 & A. benhamiae & T. verrucosum \\
\hline S8 & 7 & 3 & 14 & 14 \\
\hline S10 & 10 & 7 & 11 & 13 \\
\hline M14 & 1 & 6 & 5 & 4 \\
\hline M28 & 4 & 6 & 8 & 8 \\
\hline M35 & 5 & 0 & 5 & 6 \\
\hline M36 & 0 & 0 & 5 & 5 \\
\hline
\end{tabular}


Table 7

Genes that share $>40 \%$ identity to known keratin degradation associated peptidases in UM 880 and UM 226 genomes

\begin{tabular}{|c|c|c|c|}
\hline Family & Peptidase with $>40 \%$ identity match & UM 880 & UM 226 \\
\hline M14 & Metallocarboxypeptidase MecpA (AAB68600), TruMCPA (ABW79919) & 1 (UM880_9235) & $\begin{array}{l}2 \text { (UM226_975, } \\
\text { UM226_2139) }\end{array}$ \\
\hline \multirow[t]{2}{*}{ M28 } & TruLAP1 (AAR96058), AfuLAP1 (AAS76670) & 1 (UM880_7307) & $\begin{array}{l}2 \text { (UM226_9084, } \\
\text { UM226_9684) }\end{array}$ \\
\hline & TruLAP2 (AAS76669), AfuLAP2 (AAR96059) & 1 (UM880_4501) & $\begin{array}{l}4 \text { (UM226_5777, } \\
\text { UM226_7154, } \\
\text { UM226_3516, } \\
\text { UM226_10463) }\end{array}$ \\
\hline S08 & $\begin{array}{l}\text { Subtilase } \\
\text { McaSub1 (CAD24008), McaSub3 (CAD24010), TruSub1 (AAR11460), TruSub3 } \\
\text { (AAR11462), TruSub4 (AAR02423), TruSub5 (AAR02424), TruSub6 (AAN32713), } \\
\text { TruSub7 (AAN03635), AfuALP2 (CAB45520) }\end{array}$ & $\begin{array}{l}4 \text { (UM880_6575, } \\
\text { UM880_7425, } \\
\text { UM880_7707, } \\
\text { UM880_10609) }\end{array}$ & $\begin{array}{l}2 \text { (UM226_366, } \\
\text { UM226_11336) }\end{array}$ \\
\hline \multirow[t]{2}{*}{ S09 } & $\begin{array}{l}\text { Dipeptidyl peptidase IV } \\
\text { TruDPPIV (AAS76665), AspDPPIV (AAC34310) }\end{array}$ & 1 (UM880_10172) & 1 (UM226_84) \\
\hline & $\begin{array}{l}\text { Dipeptidyl peptidase V } \\
\text { TruDPPV (AAN03632), AfuDPPV (AAB67282) }\end{array}$ & 1 (UM880_9819) & $\begin{array}{l}3 \text { (UM226_1515, } \\
\text { UM226_2988, } \\
\text { UM226_5926) }\end{array}$ \\
\hline \multirow[t]{2}{*}{ S10 } & Serine carboxypeptidase TruSCPC (AAS76668) & 1 (UM880_11056) & 1 (UM226_4504) \\
\hline & $\begin{array}{l}\text { Serine carboxypeptidase TruSCPA (AAS76667), AfuCp1 (AAR91697), TruSCPB } \\
\text { (AAS76666), AfuCp2 (AAR96054) }\end{array}$ & $\begin{array}{l}3 \text { (UM880_2648, } \\
\text { UM880_12214, } \\
\text { UM880_7179) }\end{array}$ & $\begin{array}{l}3 \text { (UM226_5845, } \\
\text { UM226_8220, } \\
\text { UM226_9052) }\end{array}$ \\
\hline Total & & 13 & 18 \\
\hline
\end{tabular}

Table 8

Antifungal susceptibility profile of N. dimidiatum UM 880 and B. papendorfii UM 226.

\begin{tabular}{|c|c|c|c|c|c|c|c|c|}
\hline \multirow{3}{*}{ Fungi } & \multicolumn{8}{|c|}{ Antifungal Drugs, MIC $(\mu \mathrm{g} / \mathrm{mL})$} \\
\hline & \multicolumn{4}{|l|}{ Azoles } & \multicolumn{3}{|c|}{ Echinocandins } & \multirow{2}{*}{$\begin{array}{l}\text { Amphotericir } \\
\text { B }\end{array}$} \\
\hline & Posaconazole & Voriconazole & Itraconazole & Fluconazole & Ketoconazole & Anidulafungin & Caspofungin & \\
\hline $\begin{array}{l}\text { N. } \\
\text { dimidiatum } \\
\text { UM } 880\end{array}$ & 0.25 & 0.064 & 4 & 0.023 & 0.006 & 0.004 & 0.047 & $<0.002$ \\
\hline $\begin{array}{l}\text { B. } \\
\text { papendorfii } \\
\text { UM } 226\end{array}$ & 0.006 & 0.023 & 0.012 & 1 & 0.032 & 0.004 & 0.094 & 0.012 \\
\hline
\end{tabular}


Table 9

Utilisation of carbon, nitrogen, phosphorus, sulfur, and nutritional substrates on by UM 880 and UM 226

\begin{tabular}{|c|c|c|}
\hline Carbon substrates & UM 880 & UM 226 \\
\hline m-Inositol & + & + \\
\hline D-Ribose & - & + \\
\hline Adonitol & - & + \\
\hline D-Psicose & - & + \\
\hline L-proline & - & + \\
\hline L-Rhamnose & - & + \\
\hline L-arabinose & - & + \\
\hline D-xylose & - & + \\
\hline L-Lyxose & - & + \\
\hline a-Methyl-D-Galactoside & - & + \\
\hline$\beta$-Methyl-D-Glucoside & + & - \\
\hline $\mathrm{N}$-acetyl-D-glucosamine & - & + \\
\hline Dulcitol & - & + \\
\hline D-mannitol & - & + \\
\hline D-Cellobiose & - & + \\
\hline D-trehalose & - & + \\
\hline D-Melibiose & - & + \\
\hline Sucrose & - & + \\
\hline$\beta$-Methyl-D-Glucoside & - & + \\
\hline D-galactose & - & + \\
\hline Tyramine & - & + \\
\hline Maltotriose & - & + \\
\hline a-D-Lactose & - & + \\
\hline Maltose & + & + \\
\hline Lactulose & - & + \\
\hline a-D-Glucose & + & + \\
\hline D-mannose & + & + \\
\hline Sucrose & + & - \\
\hline D-Fructose & + & - \\
\hline Glycine & - & + \\
\hline Palatinose & - & + \\
\hline Pectin & - & + \\
\hline a-Cyclodextrin & - & + \\
\hline Gentiobiose & + & + \\
\hline Xylitol & - & + \\
\hline D-Arabinose & - & + \\
\hline D-Tartaric Acid & - & + \\
\hline
\end{tabular}

Page 21/32 


\begin{tabular}{|c|c|c|}
\hline Carbon substrates & UM 880 & UM 226 \\
\hline 3-O- $\beta$-D-Galactopyranosyl-D-Arabinose & - & + \\
\hline Maltitol & - & + \\
\hline L-Ornithine & - & + \\
\hline ठ-Amino Valeric Acid & - & + \\
\hline N-Acetyl-D-Glucosaminitol & - & + \\
\hline Y-Hydroxybutyric Acid & - & + \\
\hline Glycogen & + & + \\
\hline$\beta$-Hydroxybutyric Acid & - & + \\
\hline a-Keto Valeric Acid & - & + \\
\hline$\beta$-Cyclodextrin & + & + \\
\hline D-Fucose & - & + \\
\hline D-Raffinose & - & + \\
\hline a-Methyl-D-Mannoside & - & + \\
\hline$\beta$-Methyl-D-Xyloside & - & + \\
\hline Laminarin & + & + \\
\hline Stachyose & - & + \\
\hline L-Valine & - & + \\
\hline a-Methyl-D-Glucoside & - & + \\
\hline i-Erythritol & - & + \\
\hline Mannan & - & + \\
\hline ß-Methyl-D-Galactoside & - & + \\
\hline Arbutin & + & + \\
\hline D-Arabitol & - & + \\
\hline $\beta$-Methyl-D-Glucuronic Acid & - & + \\
\hline Turanose & + & + \\
\hline Y-Amino Butyric Acid & - & + \\
\hline Dextrin & + & + \\
\hline D-Melezitose & + & + \\
\hline y-Cyclodextrin & + & - \\
\hline L-Sorbose & + & - \\
\hline Sedoheptulosan & + & - \\
\hline Salicin & + & - \\
\hline Nitrogen substrates & UM 880 & UM 226 \\
\hline Uridine & + & - \\
\hline L-Leucine & + & - \\
\hline D, L-a-Amino-N-Butyric Acid & + & - \\
\hline L-Tyrosine & + & - \\
\hline L-Histidine & + & - \\
\hline Formamide & + & - \\
\hline D-Serine & + & - \\
\hline
\end{tabular}

Page 22/32 


\begin{tabular}{|c|c|c|}
\hline Carbon substrates & UM 880 & UM 226 \\
\hline L-Isoleucine & + & - \\
\hline Inosine & + & - \\
\hline Gly-Met & + & - \\
\hline Ala-Leu & + & - \\
\hline Uric Acid & + & + \\
\hline Ethylamine & + & - \\
\hline L-Aspartic Acid & + & - \\
\hline Histamine & + & - \\
\hline N-Phthaloyl-L-glutamic Acid & + & - \\
\hline Met-Ala & + & - \\
\hline Ala-Asp & + & + \\
\hline L-Serine & + & - \\
\hline Cytosine & + & - \\
\hline L-Glutamic Acid & + & - \\
\hline Ala-Thr & + & - \\
\hline Agmatine & + & - \\
\hline Glucuronamide & + & - \\
\hline N-Acetyl-D-Glucosamine & + & - \\
\hline Ammonia & + & + \\
\hline L-Phenylalanine & + & - \\
\hline L-Tryptophan & + & - \\
\hline Guanosine & + & - \\
\hline L-Proline & + & + \\
\hline$\beta$-Phenylethylamine & + & - \\
\hline Nitrite & + & - \\
\hline L-Ornithine & + & - \\
\hline Adenine & + & - \\
\hline L-Valine & + & - \\
\hline Gly-Asn & + & - \\
\hline Putrescine & + & - \\
\hline Parabanic Acid & + & - \\
\hline D-Mannosamine & + & - \\
\hline L-Alanine & + & + \\
\hline Xanthosine & + & - \\
\hline Adenosine & + & - \\
\hline Glycine & + & - \\
\hline Ala-GIn & + & - \\
\hline Ala-Glu & + & - \\
\hline Xanthine & + & - \\
\hline Y-Amino-N-Butyric Acid & + & - \\
\hline
\end{tabular}

Page 23/32 


\begin{tabular}{|c|c|c|}
\hline Carbon substrates & UM 880 & UM 226 \\
\hline L-Asparagine & + & - \\
\hline Gly-GIn & + & + \\
\hline L-Pyroglutamic Acid & + & - \\
\hline Allantoin & + & - \\
\hline L-Glutamine & + & + \\
\hline Ala-Gly & + & - \\
\hline Tyramine & + & - \\
\hline L-Arginine & + & - \\
\hline Urea & + & - \\
\hline D-Valine & - & + \\
\hline Phosphorus substrates & UM 880 & UM 226 \\
\hline O-Phospho-L-Tyrosine & + & - \\
\hline D-2-Phospho-Glyceric Acid & + & - \\
\hline D-3-Phospho-Glyceric Acid & + & - \\
\hline Phosphocreatine & + & - \\
\hline Phosphoenol Pyruvate & + & - \\
\hline Cytidine-2',3'-cyclic mono-phosphate & + & - \\
\hline Phosphoryl Choline & + & - \\
\hline Cytidine-2'-monophosphate & + & - \\
\hline D-Mannose-1-Phosphate & + & - \\
\hline 2-Deoxy-D-Glucose 6-Phosphate & + & - \\
\hline O-Phospho-D-Serine & + & - \\
\hline O-Phospho-L-Threonine & + & - \\
\hline Sulfur substrates & UM 880 & UM 226 \\
\hline Hypotaurine & + & - \\
\hline Nutritional substrates & UM 880 & UM 226 \\
\hline L-Tryptophan & + & - \\
\hline
\end{tabular}

\section{Figures}



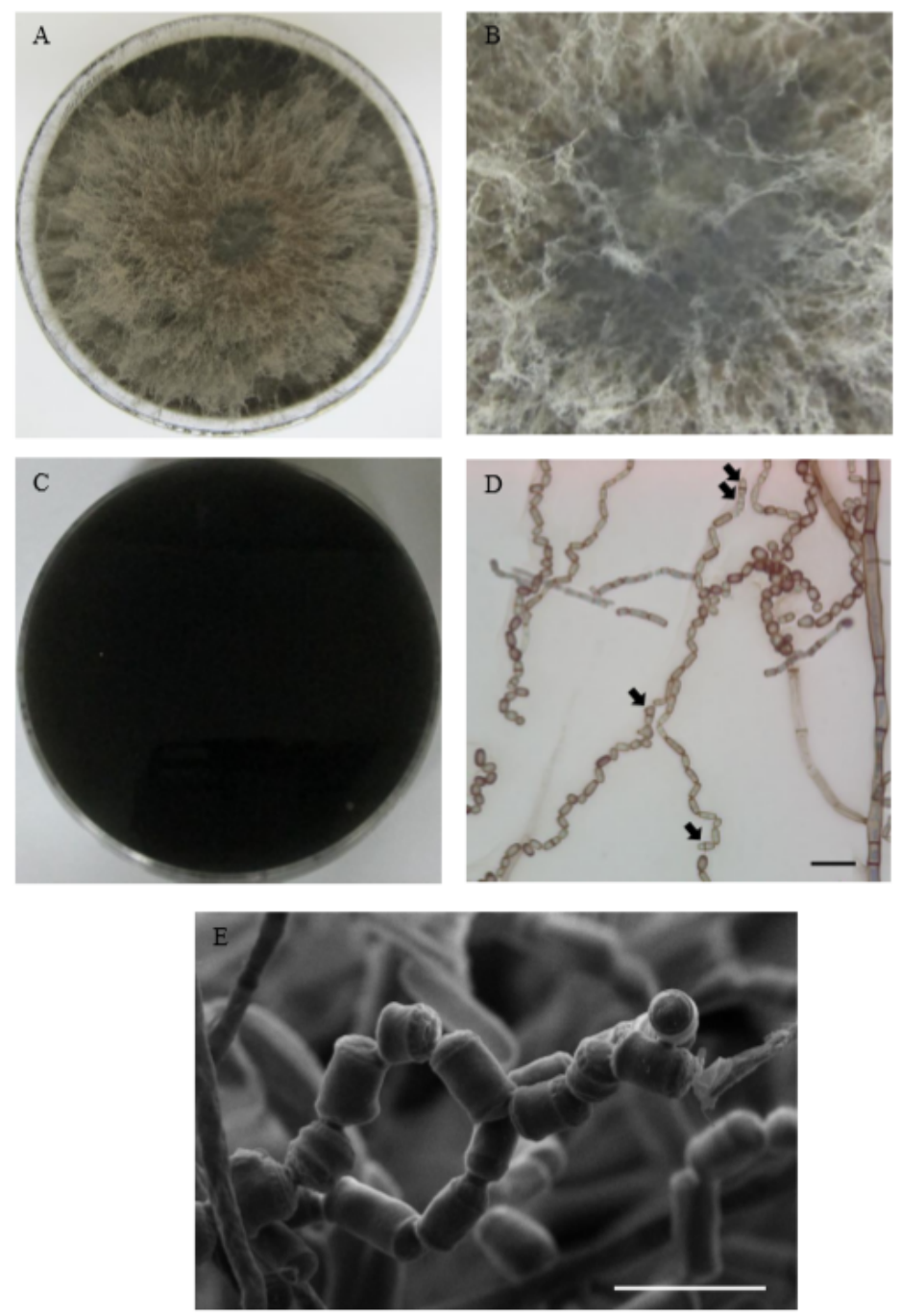

\section{Figure 1}

. Macroscopic and microscopic morphology of N. dimidiatum UM 880. The surface (A), close-up (B) and reverse (C) view of colony morphology of N. dimidiatum UM 880 after being cultured for seven days on SDA. Lactophenol cotton blue mount showing (D) typical arthroconidia of N. dimidiatum (400x magnification, bars $20 \mu \mathrm{m}$ ). Two celled conidia are also shown (arrows). Scanning electron micrograph showing (E) the arthroconidia were cylindrical with smooth to slightly verruculose walled and arranged in chains. 


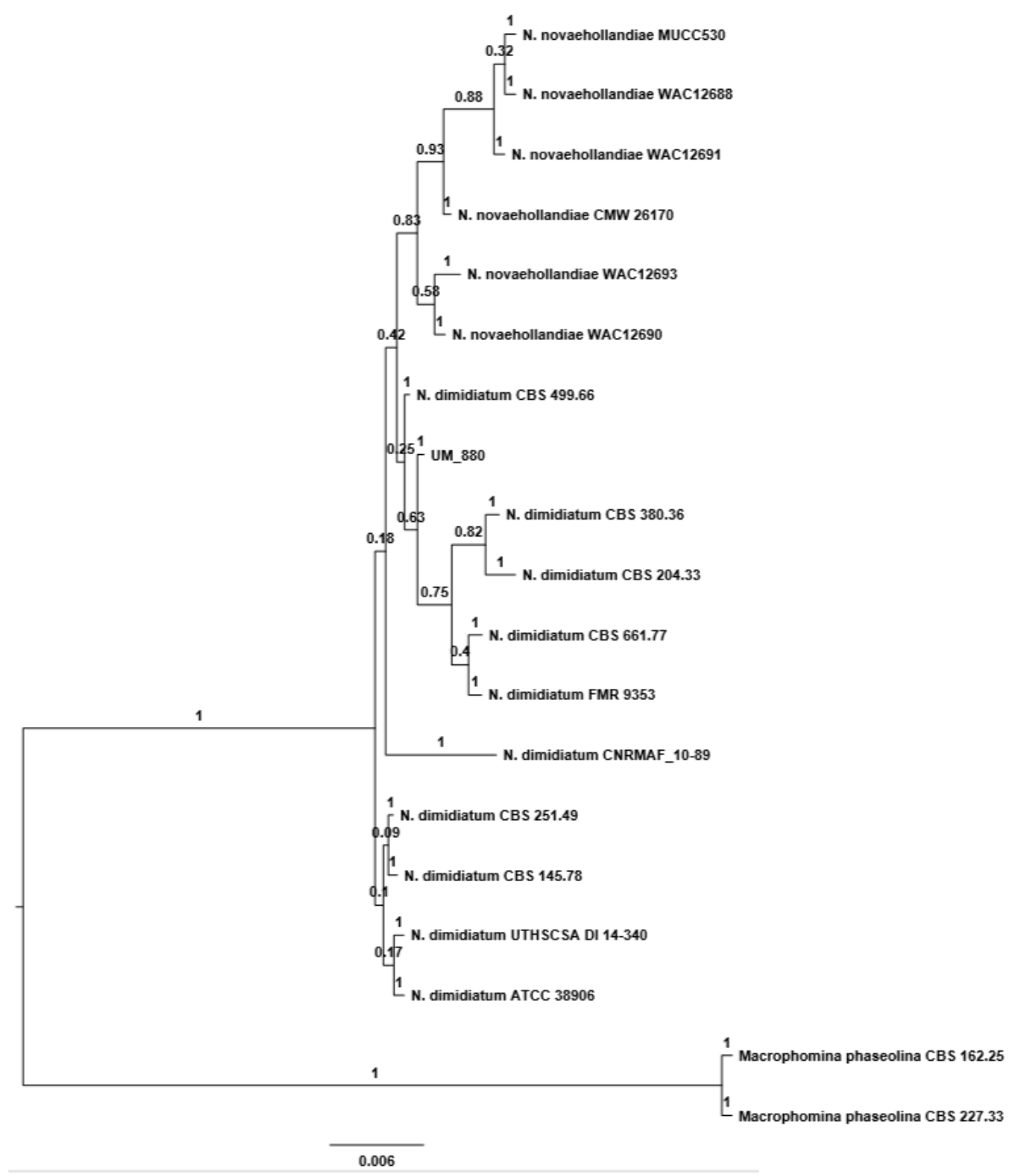

Figure 2

Bayesian phylogram generated based on combined ITS, SSU and LSU gene sequencing data. The tree is rooted with Macrophomina phaseolina CBS 162.25 and M. phaseolina CBS 227.33 as outgroup. Numbers on the nodes indicate Bayesian posterior probability based on 100 sampling frequency for a total of 150,000 generations. 


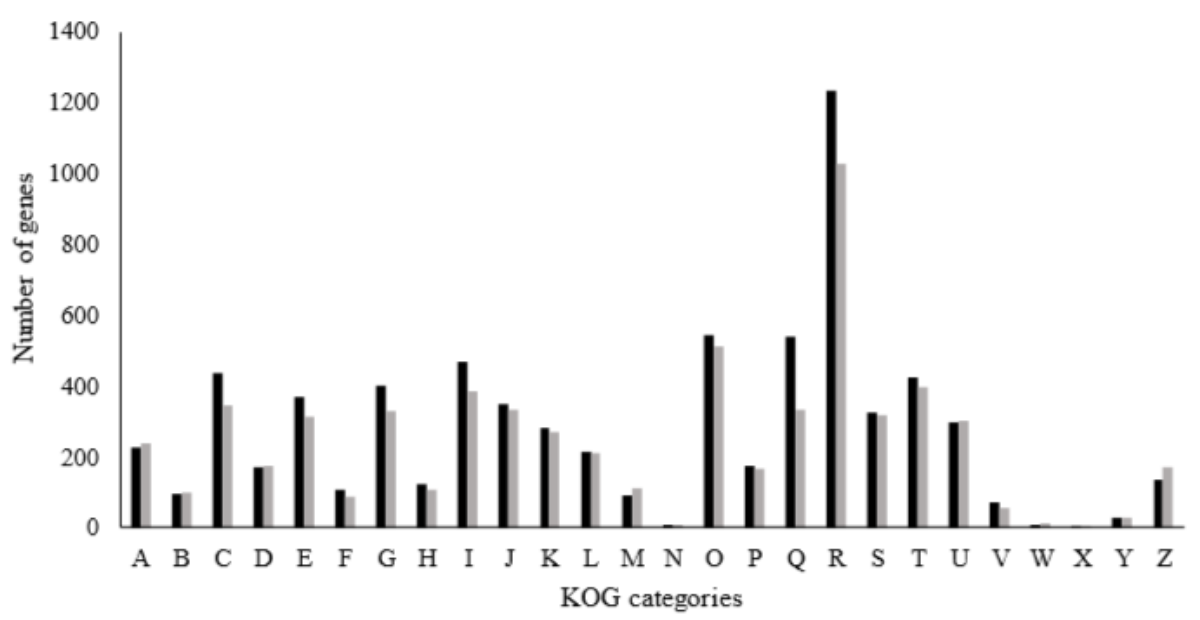

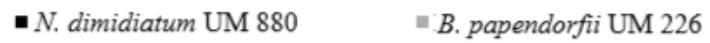

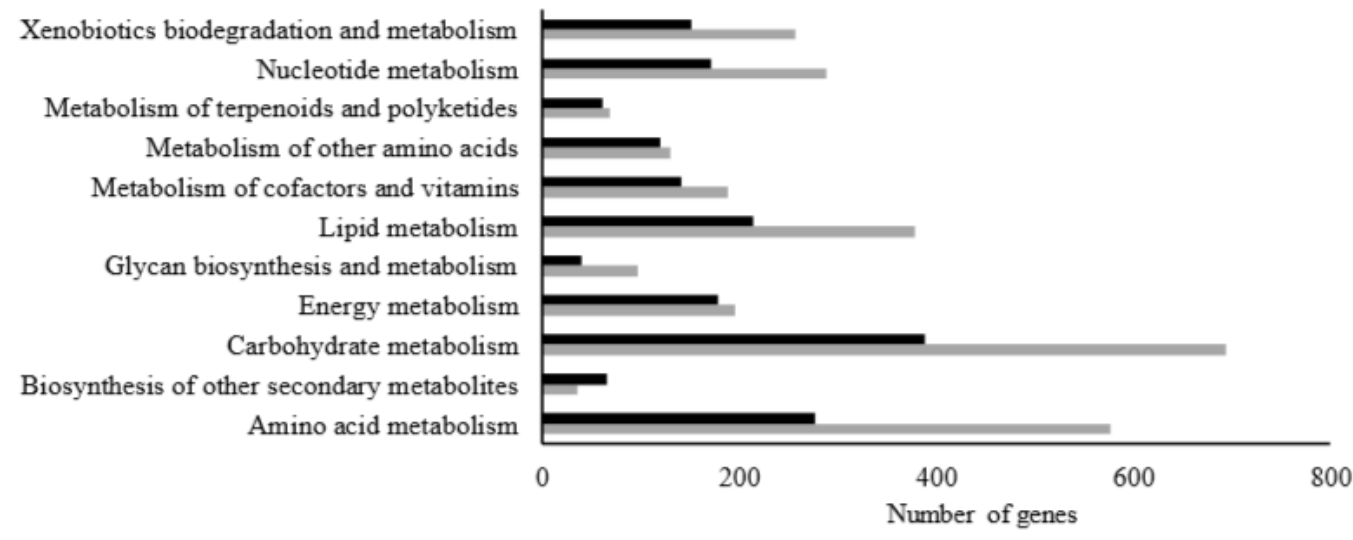

- N. dimidiatum UM 880

B. papendorfii $\mathrm{UM} 226$

Figure 3

KOG and KEGG classifications of proteins in N. dimidiatum UM 880 and B. papendorfii UM 226. (A) KOG class annotation distribution of N. dimidiatum UM 880 and B. papendorfii UM 226 genomes. A: RNA processing and modification; B: Chromatin structure and dynamics; C: Energy production and conversion; D: Cell cycle control, cell division, chromosome partitioning; E: Amino acid transport and metabolism; F: Nucleotide transport and metabolism; G: Carbohydrate transport and metabolism; H: Coenzyme transport and metabolism; I: Lipid transport and metabolism; J: Translation, ribosomal structure and biogenesis; K: Transcription; L: Replication, recombination and repair; M: Cell wall/membrane/envelope biogenesis; N: Cell motility; O: Post-translational modification, protein turnover, chaperones; P: Inorganic ion transport and metabolism; Q: Secondary metabolites biosynthesis, transport and catabolism; R: General function prediction only; S: Function unknown; T: Signal transduction mechanisms; $\mathrm{U}$ : Intracellular trafficking, secretion, and vesicular transport; V: Defense mechanisms; W: Extracellular structures; X: Unnamed protein and Z: Cytoskeleton. (B) Distribution of predicted proteins from N. dimidiatum UM 880 and B. papendorfii UM 226 genomes that involved in metabolic pathway by KEGG database. 
A
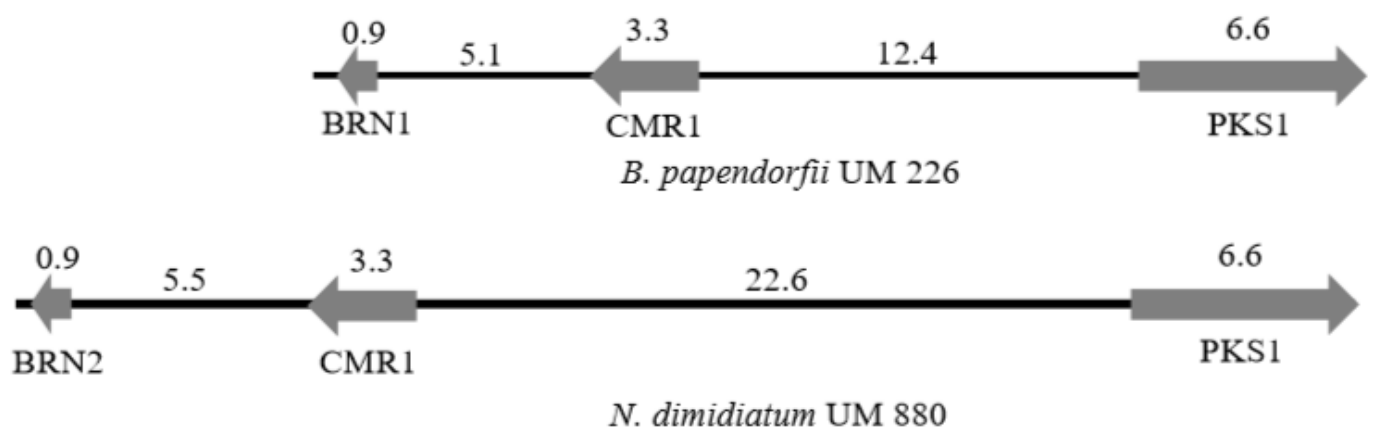

B

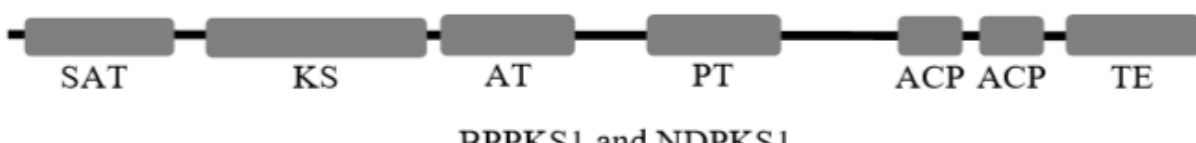

BPPKS1 and NDPKS1

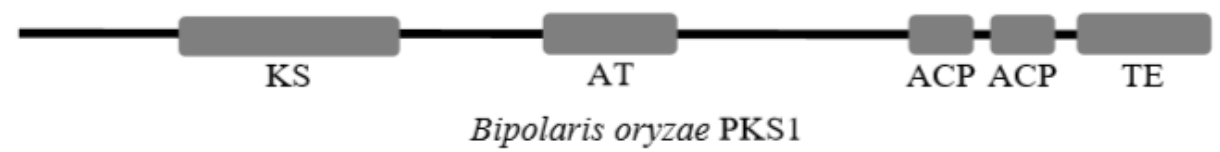

Figure 4

Melanin biosynthetic genes identified in N. dimidiatum UM 880 and B. papendorfii UM 226 genomes. (A) Schematic representation of the biosynthetic gene cluster for the PKS in N. dimidiatum UM 880 and B. papendorfii UM 226 genomes. Numbers are in kilobases. (B) Domain analysis of NDPKS1, BPPKS1, and Bipolaris oryzae PKS1. 


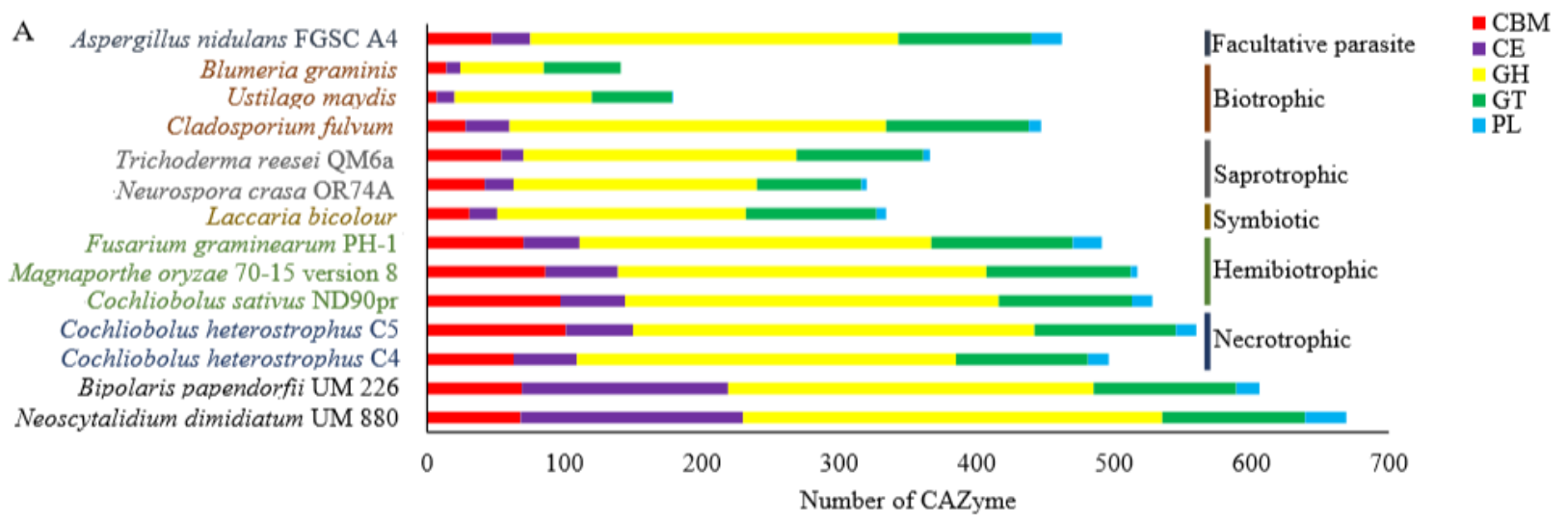

B

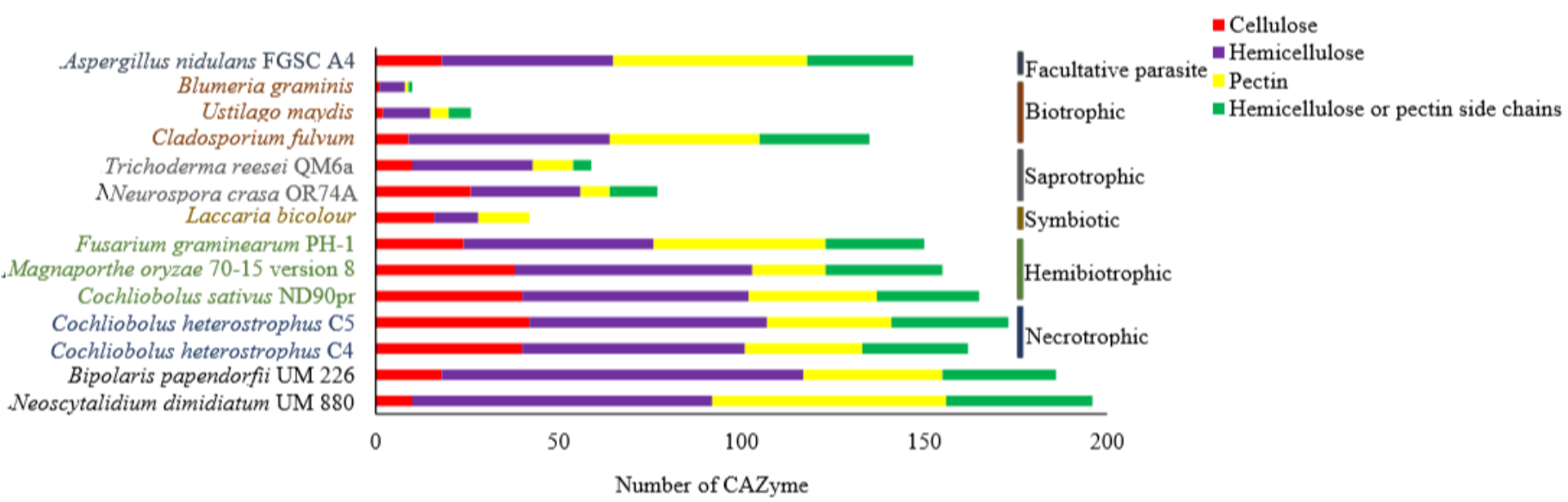

\section{Figure 5}

CAZyme class annotation distribution of N. dimidiatum UM 880 and B. papendorfii UM 226 genomes. (A) Comparison of the distribution of CAZyme catalytic domains between N. dimidiatum UM 880, B. papendorfii UM 226 and fungi from various lifestyles. AA: auxiliary activities; CBM: carbohydrate binding module; $\mathrm{CE}$ : carbohydrate esterase; $\mathrm{GH}$ : glycoside hydrolase; GT: glycosyltransferase and PL, polysaccharide lyase. (B) Comparison of the plant cell wall degrading potential from CAZyme analysis between N. dimidiatum UM 880, B. papendorfii UM 226 and fungi from various lifestyles.

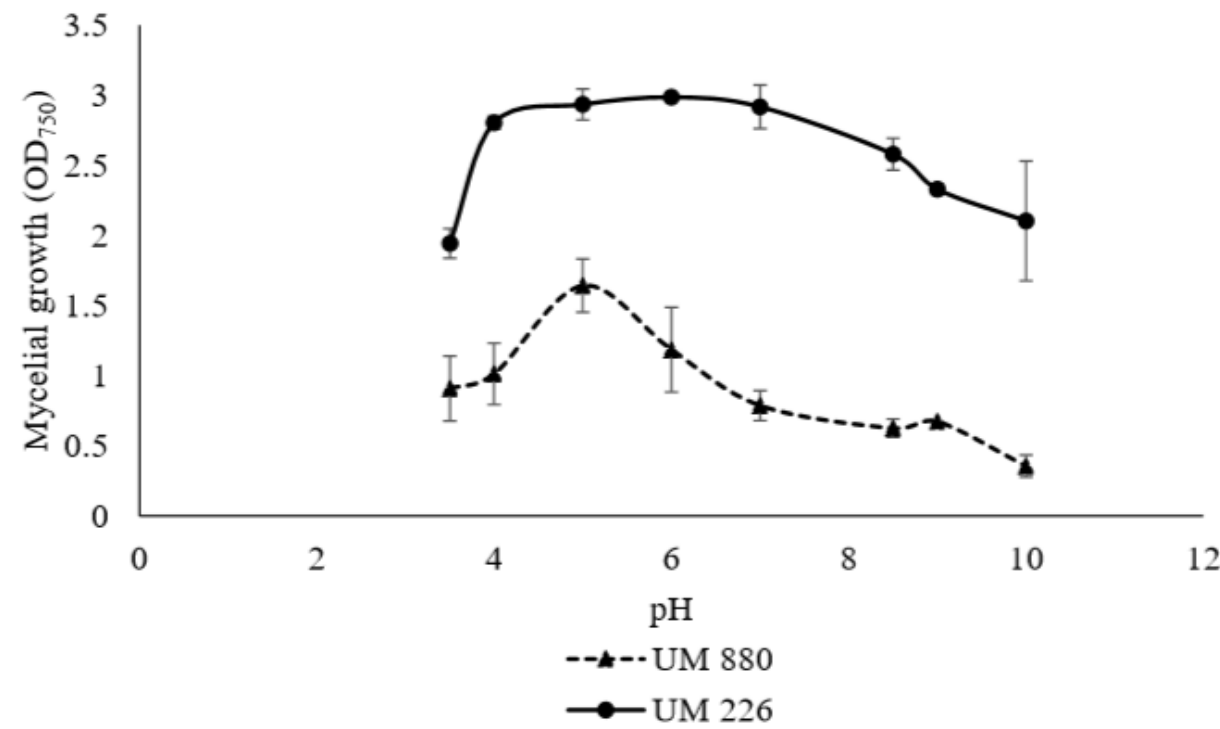


Figure 6

Mycelial growth of B. papendorfii UM 226 and N. dimidiatum UM 880 at pH 3.5-10. Results are expressed as mean \pm standard error; $\mathrm{n}=2$. The means of UM 226 and UM 880 were significantly different $(\mathrm{p}<0.05$; independent T-test) at each $\mathrm{pH}$.

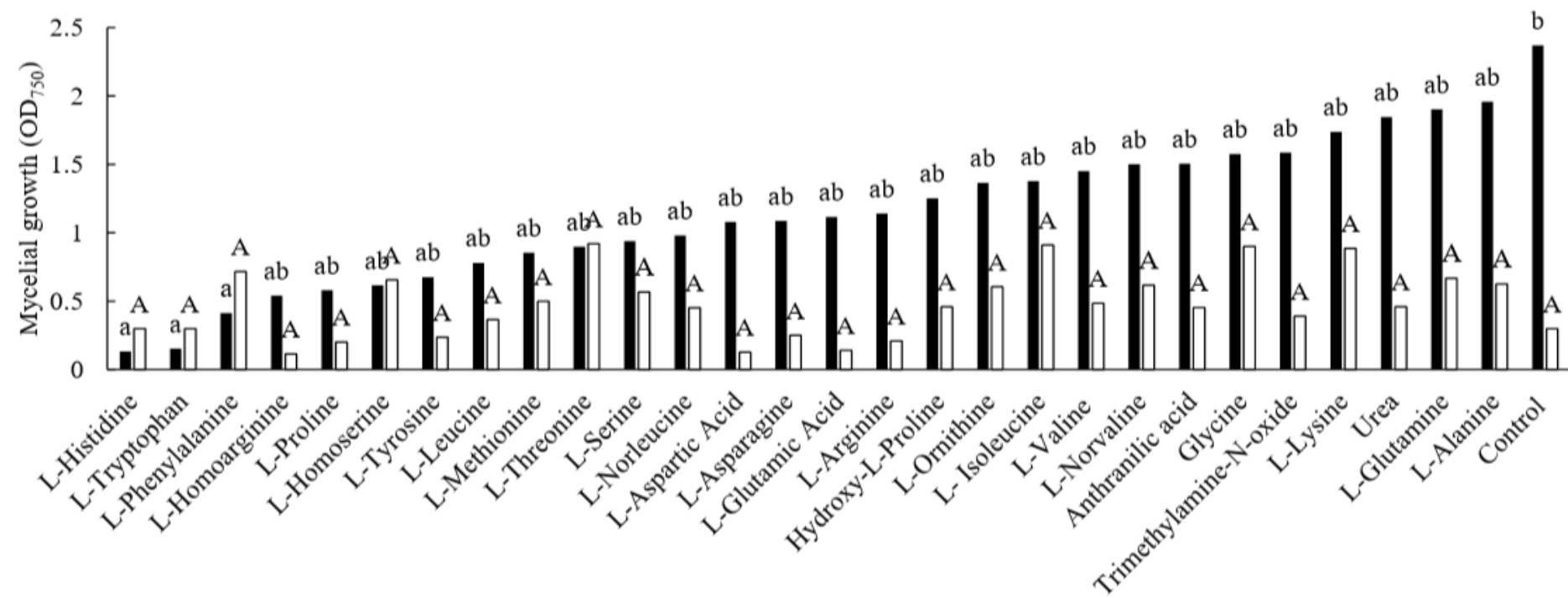

Type of nitrogen sources

-UM 226 口UM 880

Figure 7

Mycelial growth of B. papendorfii UM 226 and N. dimidiatum UM 880 in media (pH 9.5) containing various nitrogenous sources. Results are expressed as mean from two independent runs. Control represent media ( $\mathrm{pH}$ 9.5) without supplementation. ab Means for B. papendorfii UM $226 \mathrm{with}$ different lowercase letter are significantly different ( $p<0.05$; ANOVA with Tukey's post hoc test). A Means for N. dimidiatum UM 880 are not significantly different ( $\mathrm{p}<0.05$; ANOVA with Tukey's post hoc test).

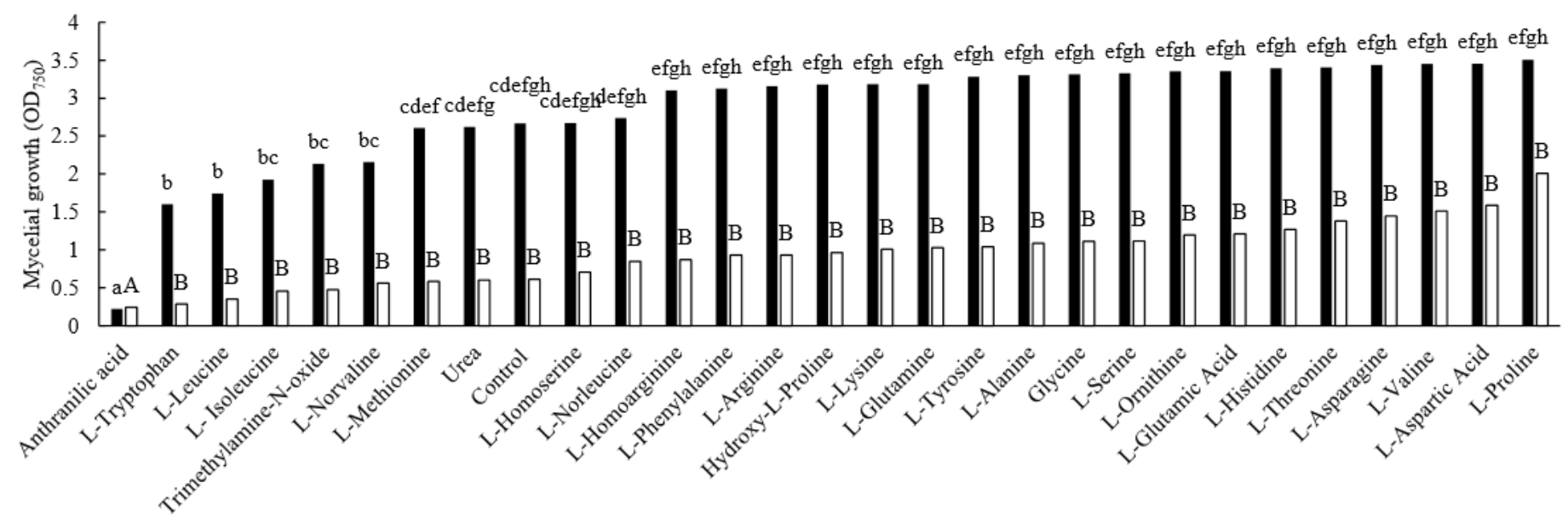

Type of nitrogenous sources

-UM 226 口UM 880

\section{Figure 8}

Mycelial growth of B. papendorfii UM 226 and N. dimidiatum UM 880 in media (pH 4.5) containing various nitrogenous sources. Results are expressed as mean from two independent runs. Control represent media ( $\mathrm{pH}$ 4.5) without supplementation. abcdefgh Means for B. papendorfii UM 226 with different lowercase letter are significantly different ( $p<0.05$; ANOVA with Tukey's post hoc test). AB Means for N. dimidiatum UM 880 with different uppercase letter are significantly different ( $p<0.05$; ANOVA with Tukey's post hoc test). 


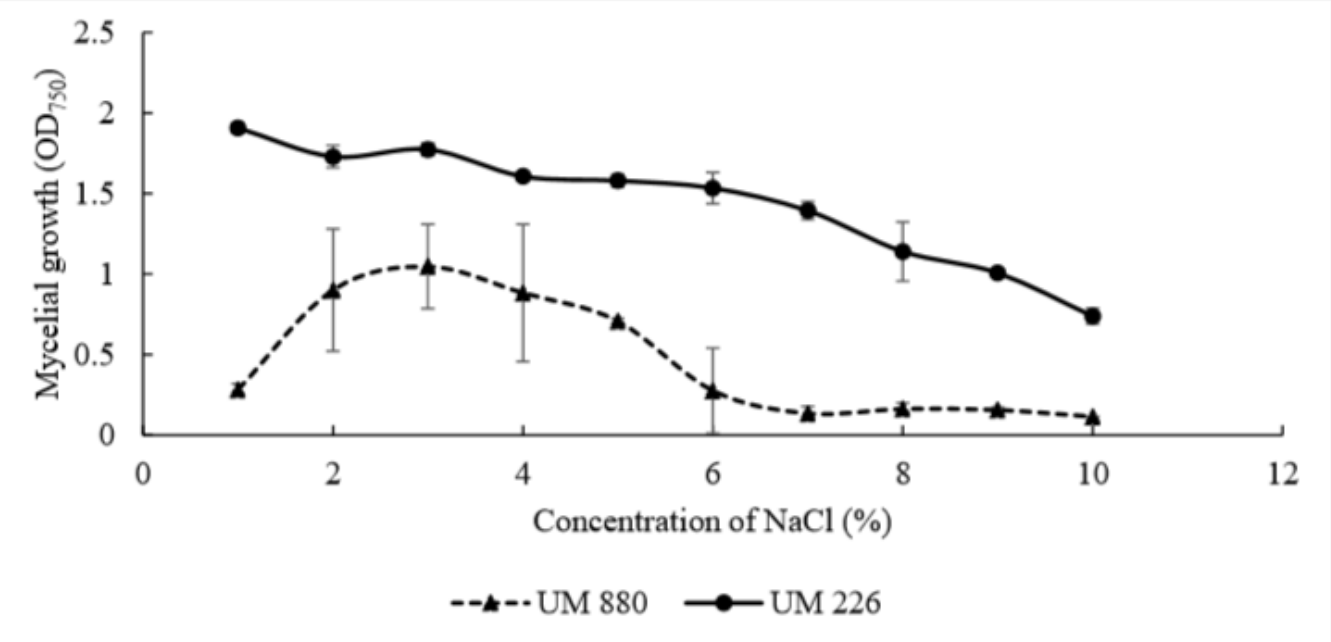

Figure 9

Mycelial growth of B. papendorfii UM 226 and N. dimidiatum UM 880 in solution containing various concentration of NaCl. Results are expressed as mean \pm standard error; $n=2$. The means of B. papendorfii UM 226 and N. dimidiatum UM 880 were significantly different ( $p<0.05$; independent T-test) at each concentration of $\mathrm{NaCl}$.

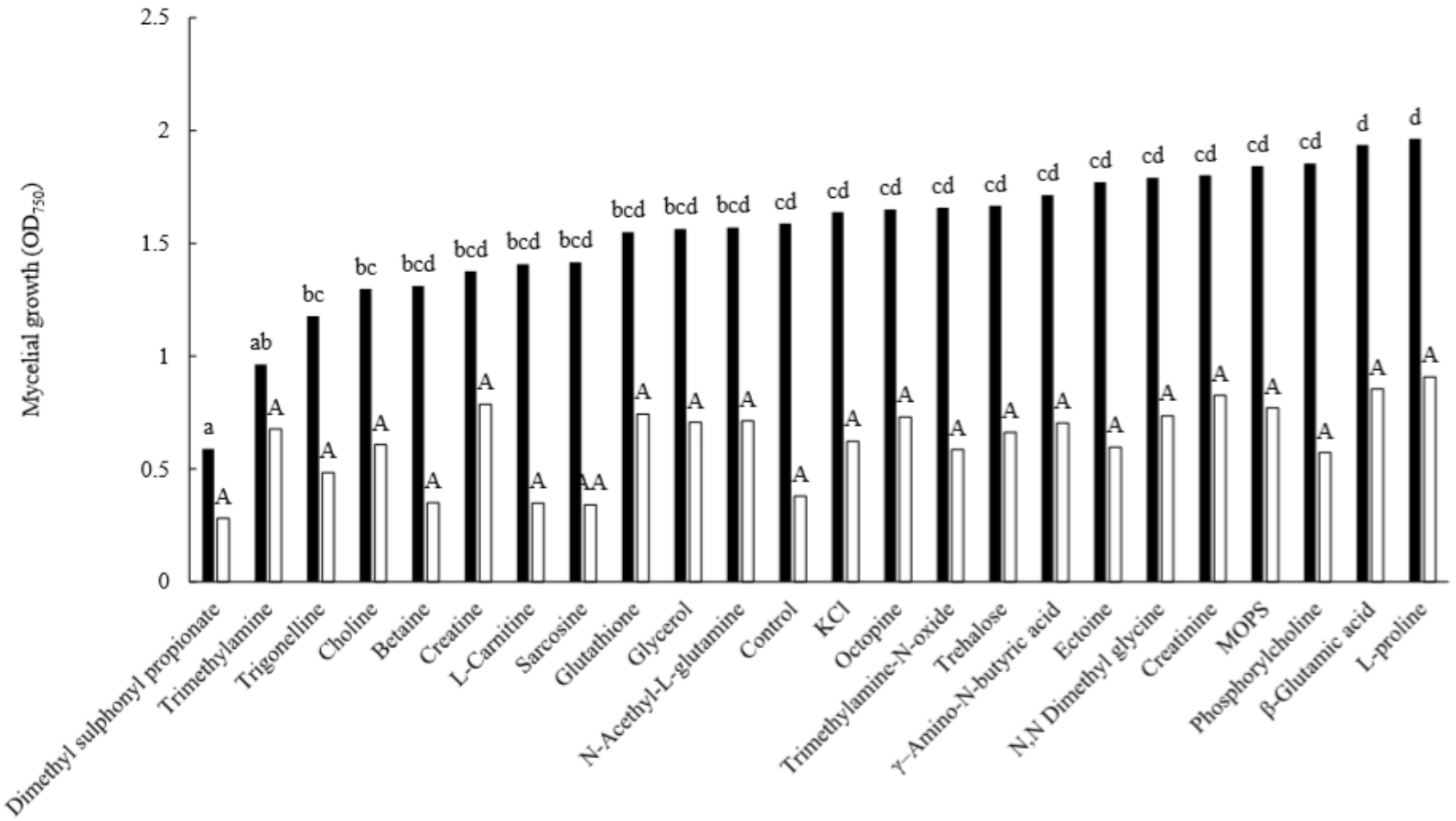

Type of osmolytes

- UM 226 口UM 880

Figure 10

Mycelial growth of B. papendorfii UM 226 and N. dimidiatum UM 880 in media containing $6 \% \mathrm{NaCl}$ and supplemented with various osmolytes. Results are expressed as mean from two independent runs. Control represent media containing $6 \% \mathrm{NaCl}$ only. 


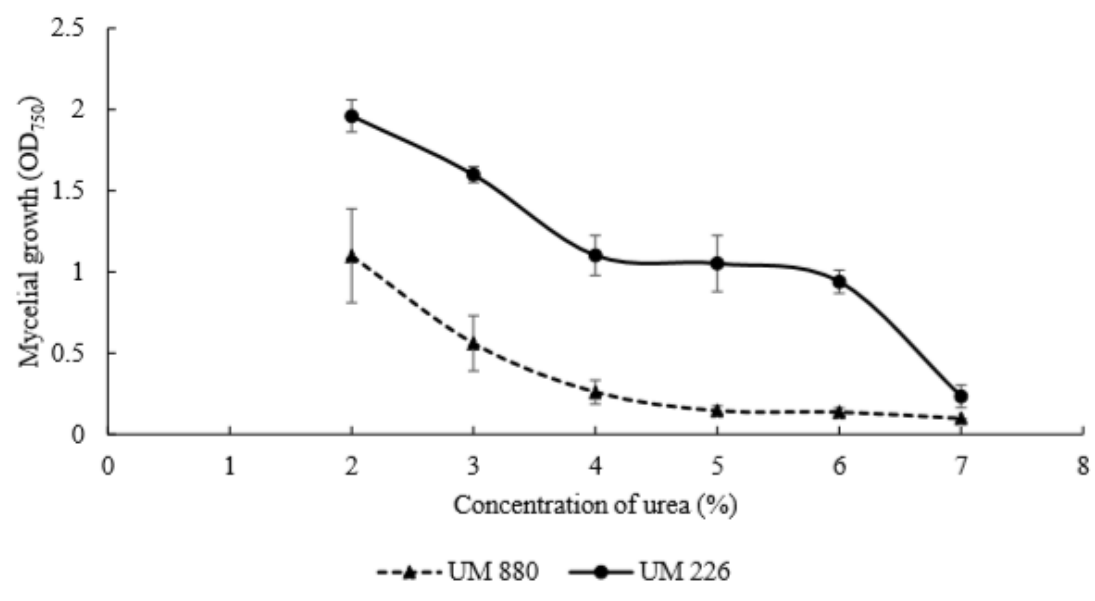

Figure 11

Mycelial growth of B. papendorfii UM 226 and N. dimidiatum UM 880 in solution containing various concentration of urea. Results are expressed as mean \pm standard error; $n=2$. The means of B. papendorfii UM 226 and $\mathrm{N}$. dimidiatum UM 880 were significantly different ( $<<0.05$; independent T-test) at $2 \%-6 \%$ of urea. The mean for both strains were not significantly different $(p>0.05)$ at $7 \%$ of urea.

\section{Supplementary Files}

This is a list of supplementary files associated with this preprint. Click to download.

- S1Table.xls

- S2Table.xIs

- S3Table.xls

- S4Table.xls.xlsx

- S5Table.xls

- S6Table.xIs.xIsx

- s7Table.xls.xlsx

- S8Table.xIs.xlsx

- S9Table.xls

- S10Table.xIs.xIsx

- S11Table.xls.xlsx

- S12Table.xls.xlsx

- S13Table.xIs.xIsx

- S14Table.xls.xlsx

- S1Fig..docx

- S2Fig..doc

- S3Fig..doc

- S4Fig..doc

- S5Fig..doc

- S6Fig..doc

- S7Fig..doc

- S8Fig..tf

- S9Fig..docx 